\title{
Integrated Variable Speed Limits Control and Ramp Metering for Bottleneck Regions on Freeway
}

\author{
Ming-hui Ma, ${ }^{1,2}$ Qing-fang Yang, ${ }^{1,2,3}$ Shi-dong Liang, ${ }^{2}$ and Zhi-lin $\mathrm{Li}^{1,2,3}$ \\ ${ }^{1}$ State Key Laboratory of Automotive Simulation and Control, Jilin University, Changchun 130022, China \\ ${ }^{2}$ College of Transportation, Jilin University, Changchun 130022, China \\ ${ }^{3}$ Jilin Province Key Laboratory of Road Traffic, College of Transportation, Jilin University, Changchun 130022, China
}

Correspondence should be addressed to Qing-fang Yang; yangqf@jlu.edu.cn

Received 5 February 2015; Revised 5 June 2015; Accepted 6 July 2015

Academic Editor: Dong Ngoduy

Copyright (C) 2015 Ming-hui Ma et al. This is an open access article distributed under the Creative Commons Attribution License, which permits unrestricted use, distribution, and reproduction in any medium, provided the original work is properly cited.

\begin{abstract}
To enhance the efficiency of the existing freeway system and therefore to mitigate traffic congestion and related problems on the freeway mainline lane-drop bottleneck region, the advanced strategy for bottleneck control is essential. This paper proposes a method that integrates variable speed limits and ramp metering for freeway bottleneck region control to relieve the chaos in bottleneck region. To this end, based on the analyses of spatial-temporal patterns of traffic flow, a macroscopic traffic flow model is extended to describe the traffic flow operating characteristic by considering the impacts of variable speed limits in mainstream bottleneck region. In addition, to achieve the goal of balancing the priority of the vehicles on mainline and on-ramp, increasing capacity, and reducing travel delay on bottleneck region, an improved control model, as well as an advanced control strategy that integrates variable speed limits and ramp metering, is developed. The proposed method is tested in simulation for a real freeway infrastructure feed and calibrates real traffic variables. The results demonstrate that the proposed method can substantially improve the traffic flow efficiency of mainline and on-ramp and enhance the quality of traffic flow at the investigated freeway mainline bottleneck.
\end{abstract}

\section{Introduction}

The steady increases of the vehicles volume and the queue length on freeway have led to efficiency loss, aggravated noise, and air pollution. Since endless expansion of road infrastructure is unpractical, because of the limitation of land resources and capital investments, it has been gradually recognized that more efficient utilization of existing freeway facility through suitable management and control strategies is essential. An increasingly important part in the field of the traffic engineering is freeway intelligent traffic control. A number of methods that have been developed in the previous designs of control strategies include control methods such as ramp metering (RM), route guidance (RG), and variable speed limits (VSL). These approaches involve neural networks, feedback control, the optimal control, and so forth. In this paper, the optimal control method is applied for design of optimal control strategies that aim to develop system optimum conditions in the freeway networks.
The bottleneck region as a danger zone is formed by the inevitable decreased number of lanes or occasional incident on the freeway networks. When the traffic flow in a region upstream of a bottleneck is more than the bottleneck capacity, the bottleneck region is activated promptly and leads to a series of corresponding traffic problems [1]. In this paper, the optimal control approach is employed for integrating VSL and ramp metering at mainline lane-drop bottleneck region.

Ramp metering methods were widely applied in freeway dynamic traffic control $[2,3]$, aimed at improving the traffic conditions by appropriately imposing restrictions on the onramp traffic flow [4-6]. Though such RM methods perform well under free-flow condition on the mainline, they are insufficient for improving the efficiency of the freeway system. Several previous studies had expressed the shortcoming of the ramp metering, in Middelham [7], Papamichail et al. $[8,9]$, and Carlson et al. [10]. There are two main weaknesses that can be summarized as follows: Firstly, the influence of the ramp metering is weak with a great number of the on-ramp 
vehicles flowing into the freeway mainline. Secondly, the effects of the ramp metering are weakened to some extent because the on-ramp supplied space is limited. Accordingly, a growing number of scholars devote themselves to the study of using other control measures on freeway network to mitigate the congestion in the freeway networks.

Another powerful control approach is variable speed limits for traffic flow on freeway mainline. The VSL is realized by displaying proper speed limit values on variable message signs in response to current traffic condition. Instead of static speed limit that is only helpful for drivers under normal traffic condition, variable speed limits are more suitable for drivers' speed choice during congestion period or inclement weather $[2,11-14]$. In previous research, there were two main purposes for the use of variable speed limits. In Abdwl-Aty et al. [15], Heydecker and Addison [16], and Zegeye et al. [17], they took the homogenization effect as the ultimate goal. On the other side, in Kejun et al. [18], Nissan and Koutsopoulosb [19], Yang et al. [20], and Yu and Abdel-Aty [21], they mainly focused on the prevention of traffic breakdown. However, ignoring the effect of the on-ramp traffic flow may result in chaos in the aggregation of that traffic flow coming from both mainline and the on-ramp [22], which could not achieve the ultimate goal of alleviating the congestion of bottleneck.

Further, either variable alone (variable speed limits or single ramp metering) is insufficiently presented above for improving the efficiency and safety on the freeway. Therefore, this paper proposes an integrated control strategy considering both elements together, ramp metering and variable speed limits, upstream of the freeway's bottleneck, aiming to improve the capacity and reduce travel delays in the bottleneck region. Integrated control strategies consider a variety of control methods $[23,24]$. In the past, several publications regarding nonlinear constrained optimal control methods to integrate ramp metering were proposed [11, 25-27], while other studies reported on the optimal integrated control applications to the merge area in freeway networks $[22,28]$. Although numerous researchers have dedicated themselves to this topic, these previously considered control methods have several disadvantages. In previous integrated control strategies, to prevent interruption of mainstream traffic near on-ramps and achieve the goal of freeway transportation system optimization, the right of on-ramp vehicles to join mainline traffic was greatly deprived and the ramp queue length was simply considered as a constraint condition. However, such control methods are not suitable for certain conditions. For example, when the traffic flow of the mainline is congested and the demands of the on-ramp are low, onramp vehicles are not allowed to travel into the mainstream until the traffic flow on the mainline is less congested. Waiting too long for on-ramp vehicles is not appropriate in such cases. Therefore, the priority of mainline traffic flow should be limited.

In this paper we propose the optimal integration of variable speed limits and ramp metering, aiming to maximize the traffic volume and minimize the average delay in freeway bottleneck regions. At the core, the proposed method is meant to enhance the efficiency of the whole traffic system by alleviating traffic congestion in bottleneck region and adjusting vehicle priority. To validate the effectiveness of the proposed control model, the result of integrated control method applied is compared with the other two cases: one case where only the variable speed limits control is adopted to manage the traffic flow upstream of the bottleneck region and the other case where no-control strategy is used for freeway network management. The contributions of this paper can be summarized as follows:

(i) We apply the optimal integrated control strategy for freeway bottleneck regions, which results in the improvement of capacity and decrease of average delay.

(ii) A novel control strategy is proposed restricting the priority for mainline traffic flow and the metering rate is calculated according to the degrees of congestion on both mainline and ramp. The more the congestion is, the more the rights of way should be given.

(iii) The bottleneck region, as defined in this paper, includes the mainline lane-drop section and the upstream affected section. The bottleneck is caused by changing the amount of lanes.

The rest of the paper is organized as follows. In Section 2, the macroscopic traffic flow model METANET is employed for traffic flow description, followed by some extensions to describe traffic flow under VSL control. An integrated control strategy for bottleneck region is developed and "the degree of congestion" is proposed to coordinate the traffic flow upstream of mainline and on-ramp in Section 3. The integrated optimal control with the goals of maximizing traffic volume and minimizing average delay is presented in Section 4, along with constraint conditions considering the limitations of application in real traffic system. The illustrative results are discussed under different control scenarios in Section 5. The conclusions and future work are summarized in Section 6.

\section{Macroscopic Traffic Flow Model}

The METANET model, as a macroscopic second-order flow model whose theory is similar to fluid mechanics that could describe the traffic flow space-time characteristic accurately, was described by Papageorgiou et al. [29, 30], Kotsialos et al. [25], Kotsialos et al. [31], and Papamichail et al. [8, 9]. This paper refers to the METANET model as the foundation model, which is extended to describe the traffic flow under variable speed limits on freeway networks.

2.1. The Original Model. The basic METANET model represents a freeway network as a directed graph whereby links of the graph are in accordance with the freeway stretches. According to consistency principle, that is, no off-ramps or on-ramps and no major geometrical changes, the mainline of the freeway network is divided into $M$ links. The nodes of the graph are situated in the major changes occurring in mainline geometrical characteristics, such as ramps or connections.

The macroscopic description of freeway traffic flow connotes the sharpness of expounded dynamic expression of 


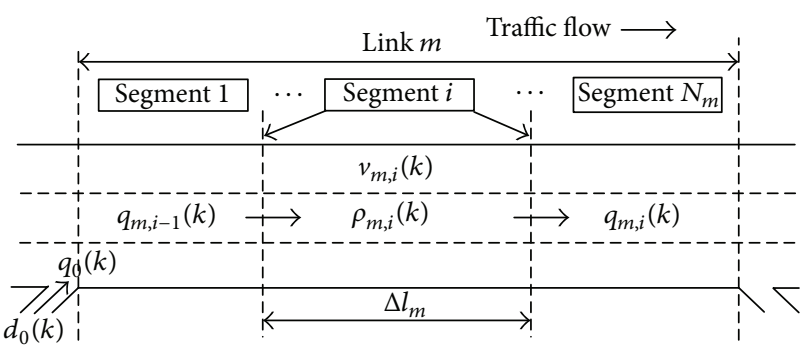

FIGURE 1: Discretised freeway link.

the traffic behavior at a certain space-time. In order to introduce the principle of METANET operation, take link $m$ as an example section. The link $m$ is divided into $N_{m}$ homogenous segments with the equal length $\Delta l_{m}$, where $\Delta l_{m}$ is given by the product of free-flow speed $v_{f, m}$ and the time interval $T$ (typically, $T=10 \mathrm{~s}$ ); see Figure 1 . For each segment $i \in\left(1,2, \ldots, N_{m}\right)$ of link $m$ at each discrete time instant $t=k T, k=0,1, \ldots, k_{p}$, where $T$ is the time instant and $k_{p}$ is the time frame, the macroscopic traffic flow parameters are defined as follows: The traffic density $\rho_{m, i}(k)$ is the number of vehicles in a single lane of segment $i$ during time period $k$. The traffic speed $v_{m, i}(k)$ is the average speed of the vehicles in segment $i$ during time period $k$. The traffic volume $q_{m, i-1}(k)$ is the number of vehicles inflowing segment $i$ during time period $k$. The traffic volume $q_{m, i}(k)$ is the number of vehicles flowing out of the segment $i$ during time period $k$.

The METANET model as the equations of traffic flow parameters of segment $i$ in link $m$ is introduced by Kotsialos et al. [31]. For implementing the description of METANET model, we review the basic METANET model in this paper here.

The outflow $q_{m, i}(k)$ equals the traffic density $\rho_{m, i}(k)$ multiplied by mean speed $v_{m, i}(k)$ and the number of lanes $\lambda_{m}$ on segment $i$ during time period $k$ :

$$
q_{m, i}(k)=\rho_{m, i}(k) v_{m, i}(k) \lambda_{m} .
$$

The traffic density $\rho_{m, i}(k+1)$ equals the previous traffic density $\rho_{m, i}(k)$ plus the variable value of density during time period $k$ :

$$
\rho_{m, i}(k+1)=\rho_{m, i}(k)+\frac{T}{\Delta l_{m} \lambda_{m}}\left[q_{m, i-1}(k)-q_{m, i}(k)\right] .
$$

The mean speed $v_{m, i}(k+1)$ during time period $k+1$ equals the previous mean speed $v_{m, i}(k)$ plus a slack movement that drivers mean to achieve an expected speed, velocity change caused by the inflow $q_{m, i-1}(k)$, and the increase (or decrease) of traffic speed due to the downstream density fluctuation leading to drivers experience of speed changed during time period $k$ :

$$
\begin{aligned}
v_{m, i}(k+1)= & v_{m, i}(k)+\frac{T}{\tau}\left\{V\left[\rho_{m, i}(k)\right]-v_{m, i}(k)\right\} \\
& +\frac{T}{\Delta l_{m}}\left[v_{m, i-1}(k)-v_{m, i}(k)\right] v_{m, i}(k) \\
& -\frac{v T}{\tau \Delta l_{m}} \frac{\rho_{m, i+1}(k)-\rho_{m, i}(k)}{\rho_{m, i}(k)+\kappa},
\end{aligned}
$$

where $\tau, v$, and $\kappa$ are constant parameters, which are determined by the traffic system, driver's behavior, geometry characteristic of link $m$, and so forth. The expected speed $V\left[\rho_{m, i}(k)\right]$ is expressed as follows:

$$
V\left[\rho_{m, i}(k)\right]=v_{f, m} \exp \left[-\frac{1}{\alpha_{m}}\left(\frac{\rho_{m, i}(k)}{\rho_{c, m}}\right)^{\alpha_{m}}\right],
$$

where $\alpha_{m}$ is the model parameter and $\rho_{c, m}$ is the critical density per lane.

The process of the origin links, which receive the demand and transport traffic flow into the freeway networks, is modeled with an elementary queue model. The queue length of the origin link $0\left(w_{0}(k+1)\right)$ is determined by the previous queue length $w_{0}(k)$, the demand $d_{0}(k)$, and the traffic flow $q_{0}(k)$ of the origin link 0 flowing into mainline during time period $k$ :

$$
w_{0}(k+1)=w_{0}(k)+T\left(d_{0}(k)-q_{0}(k)\right) .
$$

The traffic flow $q_{0}(k)$ of link 0 depends on the mainline traffic conditions and ramp metering measures. Specifically, without ramp metering, the traffic flow $q_{0}(k)$ is determined by the minimal value among the available traffic, the traffic capacity of on-ramp, and traffic flow supplied by the mainline during time period $k$ :

$$
\begin{aligned}
& q_{0}(k)=\min \left(d_{0}(k)\right. \\
& \left.\quad+\frac{w_{0}(k)}{T}, Q_{0}, Q_{0}\left(\frac{\rho_{\max , m}-\rho_{m, 1}(k)}{\rho_{\max , m}-\rho_{c, m}}\right)\right),
\end{aligned}
$$

where $\rho_{\max , m}$ is the maximal density of link $m, \rho_{m, 1}(k)$ is the density of segment $1 \mathrm{in}$ link $m$ during the period $k$, and $Q_{0}$ is the traffic capacity of on-ramp.

Otherwise, when ramp metering is applied, a proper ramp metering rate $r_{0}(k)$ is proposed. The traffic flow $\widehat{q}_{0}(k)$ of link 0 needs to consider the mainline supplied space, ramp demand, and the traffic capacity of ramp:

$$
\widehat{q}_{0}(k)=r_{0}(k) q_{0}(k),
$$

where $r_{0}(k) \in[0,1]$ is the ramp metering rate, that is, a control variable. Particularly, when the metering rate is zero $\left(r_{0}(k)=0\right)$, it expresses the fact that there is no traffic flow leaving from on-ramp. If the metering rate is $1\left(r_{0}(k)=1\right)$, it means that no ramp metering is applied and the traffic flow of ramp contains a state of self-organizing operation.

Considering the mainline velocity drop caused by ramp traffic flow in case an on-ramp existed, the velocity change is described by the term

$$
-\frac{\delta T q_{0}(k) v_{m, 1}(k)}{L_{m} \lambda_{m}\left(\rho_{m, 1}(k)+\kappa\right)},
$$

where $\delta$ is a constant parameter.

If there is a lane drop, the velocity change can be expressed as

$$
-\frac{\phi T \rho_{m, N_{m}}(k) v_{m, N_{m}}^{2}(k)\left(\lambda_{m}-\lambda_{m+1}\right)}{L_{m} \lambda_{m} \rho_{\text {crit }, m}}
$$


where $\phi$ is the model parameter and $\lambda_{m}-\lambda_{m+1}$ is the number of reducing lanes.

Equations (1) to (9) show the link $m$ traffic characteristic parameters. The traffic flow parameters of the link $m$ can be achieved by the mutually recursive equations.

The nodes connecting with the adjacent links (such as freeway junction and bifurcation) are placed between adjacent links. The node is represented as a medium connecting the input with output links here. Traffic flow enters node $n$ via a plenty of input links and is distributed to a mount of export links, which is given by the following:

$$
\begin{gathered}
Q_{n}(k)=\sum_{\mu \in I_{n}} q_{\mu, N_{\mu}}(k), \\
q_{m, 0}(k)=\beta_{n}^{m}(k) Q_{n}(k),
\end{gathered}
$$

where $I_{n}$ is a set of links entering node $n, Q_{n}(k)$ is the total traffic flow entering node $n$ during time period $k, \beta_{n}^{m}(k)$ is the turning rates, a part of traffic flow leaving the node $n$ via link $m$, and $q_{m, 0}(k)$ is the traffic flow leaving node $n$ to link $m$ during time period $k$.

When node $n$ has more than one leaving link, the upstream influence of traffic density in the last segment of the link $m$ and the downstream influence of the speed have to be considered. The downstream density $\rho_{m, N_{m+1}}(k)$ and the mean speed $v_{m, 0}(k)$ are given by the following:

$$
\begin{aligned}
\rho_{m, N_{m+1}}(k) & =\frac{\sum_{\mu \in o_{n}} \rho_{\mu, 1}^{2}(k)}{\sum_{\mu \in o_{n}} \rho_{\mu, 1}(k)}, \\
v_{m, 0}(k) & =\frac{\sum_{\mu \in I_{n}} v_{\mu, N_{\mu}}(k) q_{\mu, N_{\mu}}(k)}{\sum_{\mu \in I_{n}} q_{\mu, N_{\mu}}(k)},
\end{aligned}
$$

where $o_{n}$ is the total links leaving node $n$ and $\rho_{\mu, 1}(k)$ is the density of the first segment of link $\mu$.

2.2. Incorporate the Variable Speed Limits. The original METANET model chose the desired speed model (Equation (4)) as the transmit model input. However, under the variable speed limits control, the desired speed model presents some differences in relation to the desired speed model. These differences are mainly reflected in two aspects. First drivers desire higher speeds more in free-flow conditions. Secondly, when the traffic flow is congested, the practical traffic speed diagram fluctuates with the change of traffic flow, which is not very steady due to the drivers being constantly on and off the accelerator [32]. However, the speed curve depending on (4) is smooth, so that (4) only expresses the traffic velocity without speed limits control.

Since the original METANET model cannot express the effect of speed limits, we have to incorporate the influence of variable speed limits to freeway traffic operation by analyzing the difference between those with and those without speed limits control. Previous research often describes the influence of the variable speed limits on traffic by taking the speed limit rate that is expressed by the ratio between the limit speed and the free-flow speed $[18,33]$. There are, however, some shortcomings with this expression, which changes the whole speed-density diagram and exaggerates the effect of the speed limits. Particularly, there is an example that can help clarify this shortcoming: if the free-flow speed is $110 \mathrm{~km} / \mathrm{h}$ and the VSM displayed VSL value is $100 \mathrm{~km} / \mathrm{h}$, then it is supposed that the traffic flow and the speed are visibly reduced even when the traffic flow traveling average speed is $80 \mathrm{~km} / \mathrm{h}$. Additionally, reducing the desired speed also scales down the maximum traffic capability of the road; however, it is not reasonable to assume that the speed limit is higher than the critical speed (speeds where the traffic flow has not yet arrived at the capacity of road), according to Hegyi et al. [12].

In order to optimally introduce the variable speed limits factor, we should deeply analyze the traffic flow operating characteristics under variable speed limits control. The differences of the traffic operational mechanism that depend on whether the road traffic adopts the speed limits or not are described as follows:

(i) When the traffic is under free-flow condition and the speed limits control is adopted, the traffic flow speed is expressed by the limit speed $v_{\mathrm{vsl}}$ instead of the freeflow speed $v_{f}$, where the limit value has to be less than the free-flow speed value. Otherwise, the traffic flow follows self-organization under no special controls condition.

(ii) When traffic has gradually transited to congestion or traffic density has risen slightly above the critical density, the traffic under variable speed limits control contains a steady and regular operation due to drivers acting up to the speed limit values. The virtual speed values of the vehicles trend to uniform. On the contrary, without speed limits control on the freeway, the traffic speed values have a greater degree of dispersion.

(iii) When the traffic density approaches the jam density $\rho_{I}$, the traffic flow has to obey self-organizing operation. This is caused by the decrease of vehicle operation freedom in areas of high density traffic flow.

According to the discussion above, the desired speed variation caused by the variable speed limits control can be expressed as a function of traffic density. Specifically, if the actual traffic density is less than the critical density, the desired speed would take the minimum speed value between the speed without a speed limit control and the speed limit value as the desired speed. Otherwise, the desired speed must be equal to the speed value without VSL control because of the traffic flow remaining in a self-organization condition. Therefore, the desired speed under the VSL control is given by the following equation:

$$
\begin{aligned}
& V_{m, i}\left[\rho_{m, i}(k)\right] \\
& =\min \left(v_{f, m} \exp \left[-\frac{1}{\alpha_{m}}\left(\frac{\rho_{m, i}(k)}{\rho_{c, m}}\right)^{\alpha_{m}}\right], v_{\mathrm{vsl}, m, i}(k)\right),
\end{aligned}
$$

where $v_{\mathrm{vs}, m, i}(k)$ is the speed limit value of segment $i$ during time period $k$.

In addition, the traffic flow parameters can be obtained for both online and offline situations, through the formulas 


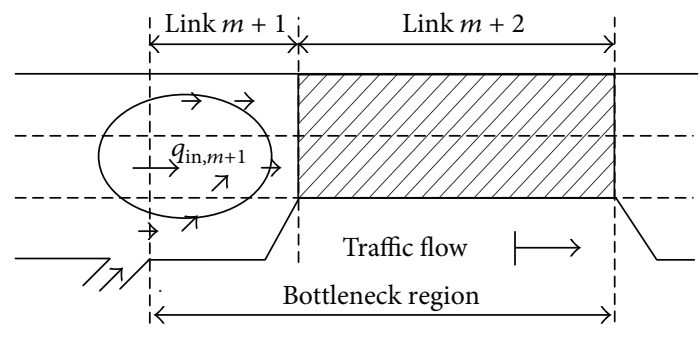

FIGURE 2: Bottleneck region.

provided in this section, and these parameters are fundamental for traffic flow description and make it possible to establish the following integrated control model.

\section{Integrated Control Algorithm Design}

Some research indicates that static speed limit control is not sufficient to relieve traffic congestion in the bottleneck regions. There are some disadvantages existing in using either the mainline control or ramp metering alone. Therefore, this section integrates the VSL and ramp metering to solve the problem of the traffic congestion caused by bottleneck regions.

3.1. Bottleneck Traffic Parameters. The mainline lane-drop bottleneck region is activated in the case that upstream outflow is higher than the traffic capacity in a given bottleneck region. In this paper, we define the bottleneck region as the region involving the bottleneck and the radiation section upstream.

Figure 2 shows link $m+1$ and link $m+2$ at the bottleneck region according to the fundamental principle of the elementary link. The links $m+2$ and $m+1$ are defined as the bottleneck and the radiation section, respectively. When the bottleneck region is activated, due to the capacity of link $m+2$ being lower than that of link $m+1$, a certain amount of chaos occurs in the link $m+1$. The vehicles from link $m+2$ and link $m+1$ influence each other, as shown in Figures 3 and 4.

In Figures 3 and $4, Q_{m+1}$ and $Q_{m+2}$ represent the traffic capacity of link $m+1$ and link $m+2, Q_{m+1}^{\prime}$ and $Q_{m+2}^{\prime}$ are the actual maximum traffic volumes, $\rho_{c, m+1}$ is the critical density of link $m+1$, and $\rho_{J, m+1}$ and $\rho_{J, m+2}$ are the jam densities of link $m+1$ and link $m+2$, respectively. When the bottleneck region is activated, the traffic flows could not travel downstream at its full load condition. Instead, a decline phenomenon concerning the traffic capacity is apparent in the bottleneck region (see Figure 4). It is quite important to emphasize the difference at the bottleneck region under critical traffic conditions as illustrated in Figures 3 and 4, which indicates the corresponding flow-density curves of the link $m+1$ and link $m+2$. In cases where the actual traffic flow $q_{m+1,1}$ is below the capacity $Q_{m+2}$, the bottleneck traffic flow remains in the free-flow, meaning that the outflow is equal to the inflow. Along with the increase of inflow, the traffic flow reaches saturated conditions during peak periods and the bottleneck outflow decreases from its capacity $Q_{m+2}$ to the virtual maximum traffic $Q_{m+2}^{\prime}$. This change is caused by the drivers experiencing serious mutual interruption in the bottleneck region, which results in the desired speed at link $m+1$ falling substantially due to the decrease in bottleneck outflow. Additionally, when the inflow of link $m+1$ is more than $Q_{m+1}^{\prime}$, queue phenomenon occurs in the link $m+1$ region, due to the shockwave effect from link $m+2$.

The above analysis of traffic characteristics that occur at bottleneck regions reflects just how complicated bottleneck traffic operations are. Thus, to build a control model to minimize the bottleneck traffic congestion problem, it is crucial to understand the specific traffic circumstances concerning link $m+2$ in the bottleneck region. Equations (13) show the evolution of traffic density and traffic volume in the link $m+2$ entrance and are shown as follows:

$$
\begin{aligned}
\rho_{m+2,1}(k) & = \begin{cases}\frac{\lambda_{m+1} \rho_{m+1, N_{m+1}}(k-1) v_{m+1, N_{m+1}}(k-1)}{\lambda_{m+2} v_{m+2,1}(k)} & \text { if } \rho_{m+1, N_{m+1}}(k)<\rho_{c, m+2} \\
\rho_{m+2,1}(k-1)+\left(\frac{q_{m+2,1}(k)-q_{m+2, N_{m+2}}(k)}{\lambda_{m+2} v_{m+2,1}(k)}\right) & \text { if } \rho_{c, m+2} \leq \rho_{m+1, N_{m+1}}(k) \leq \rho_{J, m+2},\end{cases} \\
q_{m+2,1}(k) & = \begin{cases}\min \left(v_{m+1, N_{m+1}}(k) \rho_{m+1, N_{m+1}}(k) \lambda_{m+1}, Q_{m+2}\right) & \text { if } \rho_{m+2,1}(k) \leq \rho_{c, m+2} \\
Q_{m+2}^{\prime} & \text { if } \rho_{c, m+2} \leq \rho_{m+2,1}(k),\end{cases}
\end{aligned}
$$

where $\rho_{m+1, N_{m+1}}$ is the traffic density of last segment $N_{m+2}$ of the link $m+1$.

3.2. Integrated Control Strategy. To avoid frequent deceleration and acceleration and promote the actual maximum outflow from a bottleneck region, the regional control is placed upstream of the bottleneck region. There is an on-ramp next to the controlled region and the outflow of this on-ramp also impacts the bottleneck traffic. Therefore, to achieve a more perfect control effect and solve traffic issues in bottleneck region, the traffic problems of both the mainline bottleneck and intersection $B$ have to be considered during the integrated control process. Since the main cause of the traffic problem in a mainline bottleneck is that the outflow of upstream is higher than the bottleneck capacity, it is quite essential to control the outflow of upstream traffic 


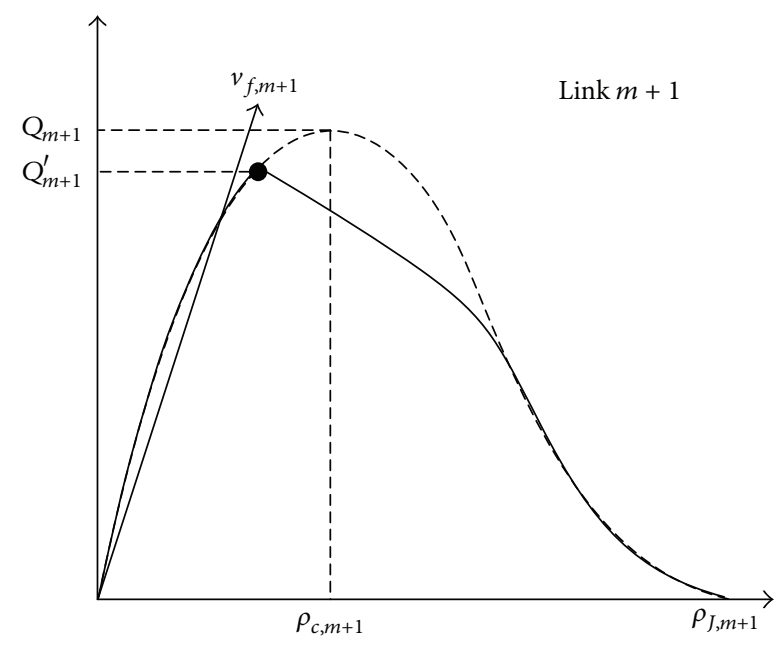

Figure 3: Flow-density diagram of the link $m+1$.

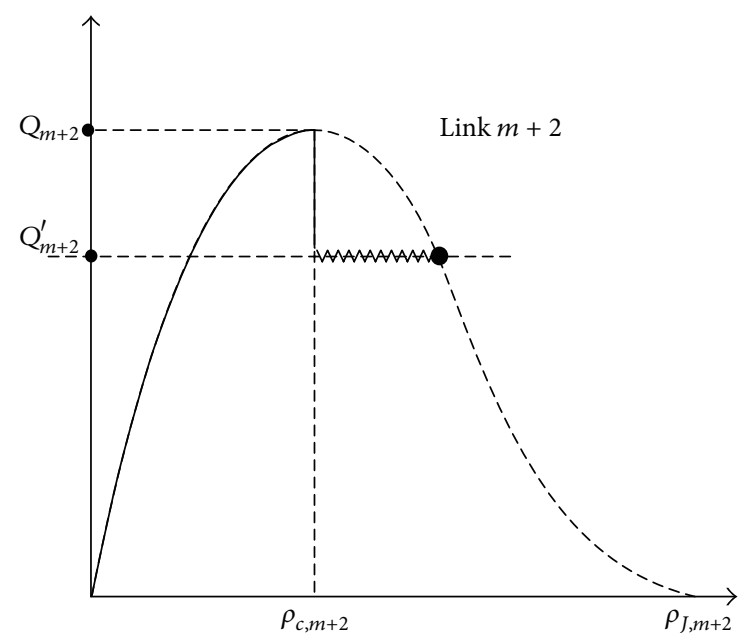

FIGURE 4: Flow-density diagram of the link $m+2$.

that consists of the traffic flow coming from the upstream mainline combined with that of the on-ramp traffic volume.

Since the bottleneck inflow is adjusted by controlling upstream outflow, it is crucial to understand the traffic element parameters of the bottleneck upstream link $m+$ 1 in order to ensure the rationality of bottleneck outflow and resolve the traffic problem within the bottleneck region. Figure 5 depicts the upstream link $m$, which is divided into three areas depending on the consistency of traffic operations and can be described as adjusted area $A$, VSL control area, and intersection $B$.

Traffic nodes were placed at the ends of link $m$ to transmit information about the amount of the traffic input links and distribute it downstream to link $m+1$. The traffic flow entering link $m q_{\text {in, } m}(k)$ equals the difference between the upstream mainline outflow $q_{\text {out }, m-1}(k)$ and the leaving flow $q_{s_{m-1}}$ of offramp $s_{m-1}$ during the time period $k$, which can be determined using the following equation:

$$
q_{\mathrm{in}, m}(k)=q_{\mathrm{out}, m-1}(k)-q_{s_{m-1}}(k) .
$$

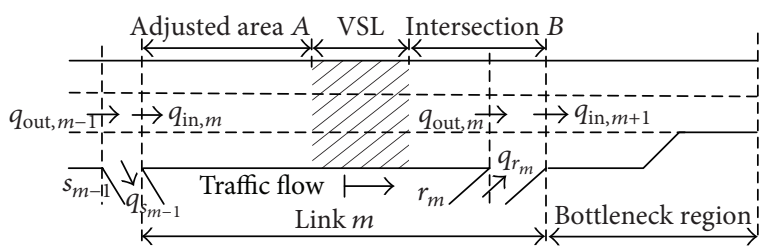

FiguRE 5: Upstream of bottleneck region.

The traffic volume leaving link $m$ is determined as follows:

$$
q_{\text {in, } m+1}(k)=q_{\text {out }, m}(k)+q_{r_{m}}(k),
$$

where $q_{\text {in }, m+1}(k)$ is the outflow of link $m, q_{\text {out }, m}(k)$ is the traffic flow leaving from the mainline link $m$, and $q_{r_{m}}(k)$ is the traffic volume derived from the on-ramp.

Since the traffic conditions contain the amount of traffic congestion at peak hours, the actual bottleneck maximum traffic volume has decreased. This paper uses an integrated control approach that involves both the variable speed limits and ramp metering to adjust the traffic inflow upstream of the bottleneck region. The integrated control approach adopts the releasing rates considering both variable speed limits and ramp metering to regulate the outflow into downstream links. The releasing rates can be achieved by the real-time degree of the congestion in the on-ramp and upstream of the bottleneck region. This model conforms better to the actual demands of the traffic graphs.

The core of the integrated control model is the reasonable release of the mainline and on-ramp traffic volume depending on the degree of congestion on the mainline and the onramp, defined as $\beta_{m}$ and $\beta_{r_{m}}$, respectively, and given by the following equations:

$$
\begin{aligned}
\beta_{m}(k) & =\frac{\sum_{k=1}^{k_{p}-1} \sum_{i=1}^{N_{m}-1} \lambda_{m} \rho_{m, i}(k) \Delta l_{m}}{\rho_{J, m}(k) L_{m} \lambda_{m}}, \\
\beta_{r_{m}} & =\frac{\sum_{k=1}^{k_{p}-1} \sum_{j=1}^{N_{r_{m}}-1} \lambda_{r_{m}} \rho_{r_{m}, j}(k) \Delta l_{r_{m}}}{\rho_{J, r_{m}}(k) L_{r_{m}} \lambda_{r_{m}}},
\end{aligned}
$$

where $j \in\left(1,2, \ldots, N_{n}\right)$ is any segment of the on-ramp $r_{m}, \lambda_{r_{m}}$ is the number of on-ramp lanes, $\rho_{r_{m}, j}(k)$ is the traffic density of segment $j, \Delta l_{r_{m}}$ is the length of segment $j, \rho_{r_{m}}(k)$ is the traffic density of on-ramp, and $L_{m}$ and $L_{r_{m}}$ are the lengths of the mainline and on-ramp, respectively.

Depending on the degree of congestion, more right should be given to either the mainline or on-ramp. Considering the congestion index proposed above, the numbers of discharging vehicles for mainline and ramp can be calculated, respectively, by using the following equations:

$$
\begin{gathered}
N_{m, N_{m}}(k)=\frac{q_{m+1,1}(k) \beta_{m} T}{\left(\beta_{m}+\beta_{r_{m}}\right)}, \\
N_{r_{m}, N_{r_{m}}}(k)=\frac{q_{m+1,1}(k) \beta_{r_{m}} T \varepsilon}{\left(\beta_{m}+\beta_{r_{m}}\right)},
\end{gathered}
$$


where $N_{m, N_{m}}(k)$ and $N_{r_{m}, N_{r_{m}}}(k)$ are the numbers of discharging vehicles for the last segment of the mainline and ramp, respectively, calculated by the degree of the congestion during the time period $k$, and $\varepsilon \in[0,1]$ is the model parameter determined by the demands of actual ramp traffic.

The number of vehicles, $N_{m+1,1}(k)$, entering the downstream link $m+1$ is expressed by the number of vehicles leaving the upstream link $m$ and entering the mainline link $m$ from the on-ramp, which can be given by the equation

$$
N_{m+1,1}(k)=N_{m, N_{m}}(k)+N_{r_{m}, N_{r_{m}}}(k),
$$

where $N_{m+1,1}(k) / T$ is below the capacity of link $m$ during time period $k$. In (18), the numbers of vehicles $N_{m, N_{m}}(k)$ and $N_{r_{m}, N_{r_{m}}}(k)$ can also be expressed by

$$
\begin{aligned}
N_{m, N_{m}}(k) & =\rho_{\mathrm{vsl}, m}(k) v_{\mathrm{vsl}, m}(k) \lambda_{m} T, \\
N_{r_{m}, N_{r_{m}}}(k) & =q_{r_{m}, N_{r_{m}}}(k) \delta_{r_{m}} T,
\end{aligned}
$$

where $v_{\mathrm{vsl}, m}(k)$ is the speed limit value in the mainline control region, $\rho_{\mathrm{vsl}, m}(k)$ is traffic density of the mainline control region, and $\delta_{r_{m}}$ is the metering rate of on-ramp. According to (19), the VSL values $v_{\mathrm{vsl}, m}(k)$ and the on-ramp release rate during time period $k$ can be achieved.

When the traffic condition turns congested on the freeway mainline, the queue phenomena will be apparent in mainline area $A$ and on-ramp $r_{m}$. The number of the queue vehicles $w_{r_{m}}(k+1)$ in the on-ramp $r_{m}$ could be described as follows:

$$
w_{r_{m}}(k+1)=w_{r_{m}}(k)+T\left[d_{r_{m}, 1}(k)-q_{r_{m}, N_{r_{m}}}(k)\right] .
$$

We expressed the difference of mainstream link $m$ and on-ramp $r_{m}$ under the coordination control as compared to regular links by using (21) and (22) to elaborate the difference of the traffic leaving from link $m$. Based on the integrated control strategy, in which the metering rate and VSL values are determined by the degree of congestion, the outflows of mainline link $m$ and on-ramp $r_{m}$ are specifically expressed depending on the difference of traffic density of the link $m$. When the actual traffic density of mainline link $m$ is far below the critical density, the traffic contains the self-organizing operation, because the mainline and on-ramp flow is not limited. The outflow of mainline traffic is freedom, while the outflow of on-ramp traffic is defined as the minimum between the mainline supplied traffic space and the outflow provided by the on-ramp. If the mainline traffic density reaches the critical density, the mainline VSL control is activated and the mainline outflow is equal to the minimum of the outflow upstream, link $m$ capacity, and the releasing traffic of the VSL control region, while the outflow of onramp depends on the minimum of the queue vehicles, the on-ramp capacity, and the on-ramp releasing traffic. Hence, the outflow of the mainline link $m$ is given by the following equation:

$$
q_{\text {out }, m}(k)= \begin{cases}v_{m, N_{m}}(k) \rho_{m, N_{m}}(k) \lambda_{m} & \text { if } \rho_{m, N_{m}}(k) \leq \rho_{c, m} \\ \min \left(q_{\text {out }, m-1}(k)-q_{s_{m-1}}(k),(1-\eta) Q_{m}, \frac{N_{m}(k)}{T}\right) & \text { if } \rho_{m, N_{m}}(k)>\rho_{c, m},\end{cases}
$$

where $v_{m, N_{m}}(k)$ is average speed of the last segment $N_{m}$ of link $m$ during time period $k$. Similarly, the on-ramp $r_{m}$ outflow is given by the following equation:

$$
q_{r_{m}}(k)= \begin{cases}\min \left(\rho_{r_{m}, N_{r_{m}}}(k) v_{r_{m}, N_{r_{m}}}(k) \lambda_{r_{m}}, Q_{c, m}-v_{f, m}(k) \rho_{m, N_{m}}(k) \lambda_{m}\right) & \text { if } \rho_{m, N_{m}}(k) \leq \rho_{c, m} \\ \min \left(d_{r_{m}}(k)+\frac{w_{r_{m}}(k)}{T}, Q_{r_{m}}, \frac{N_{r_{m}}(k)}{T}\right) & \text { if } \rho_{m, N_{m}}(k)>\rho_{c, m},\end{cases}
$$

where $Q_{r_{m}}$ is the capacity of on-ramp $r_{m}$. Conservation equation (2) is replaced by conservation equation (23) that can be described as follows:

$$
\begin{aligned}
& \rho_{m_{B}, i}(k)=\rho_{m, i}(k-1)+\frac{T}{\Delta l_{m} \lambda_{m}}\left[q_{m, i-1}(k-1)\right. \\
& \left.-q_{m, i}(k-1)+q_{r_{m}, N_{r_{m}}}(k)\right] .
\end{aligned}
$$

\section{The Integrated Optimal Control Problem}

4.1. Optimization Objective. Previously, the ultimate goal of either minimizing the total travel time (TTT) or maximizing the total traffic volume (TTV) was usually applied to the model predictive control algorithm for freeway networks. Nevertheless, taking only the minimal TTT as the optimal control goal keeps the traffic density relatively low, reducing traffic volume to enhance traffic speed. When the traffic 
density remains less than the critical density, the control is effective. However, when the traffic demand increases beyond the critical density during peak periods, a serious queuing phenomenon in the upstream of control area would occur in an attempt to achieve the minimal TTT. Conversely, an approach aiming only to maximize TTV is inconsistent with the former control method aiming at minimal TTT in that it seeks to increase the traffic volume, which leads to higher traffic density and lower speeds. Accordingly, this paper mainly considers the basic mainline, on-ramp, and the bottleneck regions. However, choosing the TTT as the objective might result in ignoring on-ramp travel time because the length of on-ramp is relatively short compared with mainline. Therefore, to improve the level of service and traffic efficiency, as well as balancing the access rights of vehicles from upstream mainline and on-ramp, we selected both the TTV and the total traffic delay (TTD) as the optimal control goals.

Since the optimum integrated control aims at achieving the goal that solves the bottleneck region's congestion problems by adjusting the releasing rates of mainline and on-ramp outflow upstream, the traffic operating situations of the mainline and on-ramp have to be considered in the established integrated control model.

The total traffic volume (TTV) is described as follows:

$$
\operatorname{TTV}=T \sum_{k=1}^{k_{p}-1} \rho_{m+1, N_{m+1}}(k) v_{m+1, N_{m+1}}(k),
$$

where $\rho_{r_{m}, j}(k)$ is the traffic density in a segment $j$ of the onramp $r_{m}$ during time period $k$. The total traffic delay (TTD) is expressed as follows:

$$
\begin{aligned}
& \mathrm{TTD}=T \sum_{k=1}^{k_{p}-1} \sum_{i=1}^{N_{m}-1}\left(\Delta l_{m} \rho_{m, i}(k) \lambda_{m}-\frac{\Delta l_{m}}{v_{f, m}} q_{m, i}(k)\right) \\
& +T \sum_{k=1}^{k_{p}-1} \sum_{j=1}^{N_{r_{m}}-1}\left(\Delta l_{r_{m}} \rho_{r_{m}, j}(k) \lambda_{r_{m}}-\frac{\Delta l_{r_{m}}}{v_{f, r_{m}}} q_{r_{m}, j}(k)\right) \\
& +T \sum_{k=1}^{k_{p}-1} \sum_{i=1}^{N_{m+1}-1}\left(\Delta l_{m+1} \rho_{m+1, i}(k) \lambda_{m+1}\right. \\
& \left.-\frac{\Delta l_{m+1}}{v_{f, m+1}} q_{m+1, i}(k)\right),
\end{aligned}
$$

where $v_{f, r_{m}}$ is the free speed of on-ramp $r_{m}$. The TTV and the TTD are both taken as objective functions in the integrated control model. Since the two parts of the objective function have different dimensions, we introduced two parameters, $\alpha_{V}$ and $\alpha_{D}$, for the sake of balancing the influences of the two basic models with the final objectives of the integrated control. Hence, the optimal goal of the integrated control model is described by combining the TTV model and the TTD model as follows:

$$
J=\alpha_{D} \mathrm{TTD}-\alpha_{V} \mathrm{TTV}
$$

Through the discussion above, the nonlinear macroscopic traffic space-time model describing a freeway network can be expressed as

$$
\begin{aligned}
x(k+1) & =f[x(k), \mu(k), k] \\
x(0) & =x_{0},
\end{aligned}
$$

where $x$ and $\mu$ are the state variable and control variable, respectively. In (27), the state variables include traffic speed and density, while the control variables include the variable speed limit value and discharge rate.

4.2. Constraint Conditions. In order to ensure that the integrated strategy is reasonable, several factors (such as the traffic safety, the obedience of the drivers for the VSL values) have to be considered in the constraint conditions as follows:

(1) When the mainline traffic VSL control is adopted, the maximum VSL values must be below the safety speed of traffic, which is expressed as follows: $v_{\mathrm{vsl}, m, \max }(k) \leq$ $V_{m, \max }$.

(2) To enhance the obedience of drivers, the VSL value has to ensure that the difference value between the adjacent time intervals cannot be greater than the maximum driver obeyed speed, which can be expressed as follows: $\left|v_{\mathrm{vsl}, m, \text { max }}(k)-v_{\mathrm{vsl}, m, \text { max }}(k-1)\right| \leq$ $\Delta V_{\text {max,diff-time }}$.

(3) The VSL value should be higher than the minimum speed of the traffic flow operating on the road in order to maintain traffic flow with a reasonably low speed, which could be described as follows: $v_{\mathrm{vsl}, m, \min }(k) \geq$ $V_{m, \min }$.

\section{A Benchmark Test}

5.1. Experiment Design. The integrated control strategy can be tested by microscopic traffic simulation software. We adopted three cases to check the efficiency and rationality of the integrated control strategy, which are no-control, mainline VSL control, and integrated control. More specifically, the settings of the above schemes are designed as follows.

(i) The case of no-control (without using either the VSL or ramp metering) is represented first and is defined as case 1 .

(ii) Case 2 describes only adopting the mainline VSL control method which is the mainline control presented in (26) while there is no ramp metering.

(iii) Case 3 takes the integrated control strategy to adjust the mainline and on-ramp traffic flow to solve the traffic congestion problem in bottleneck regions.

There are some influences of the VSL region position that control the effect of the bottleneck congestion problem. For instance, if the location of the VSL control region is located far from the problematic region, the VSL would be less effective. On the other hand, if the control region and the problem region are too close, the bottleneck congestion 


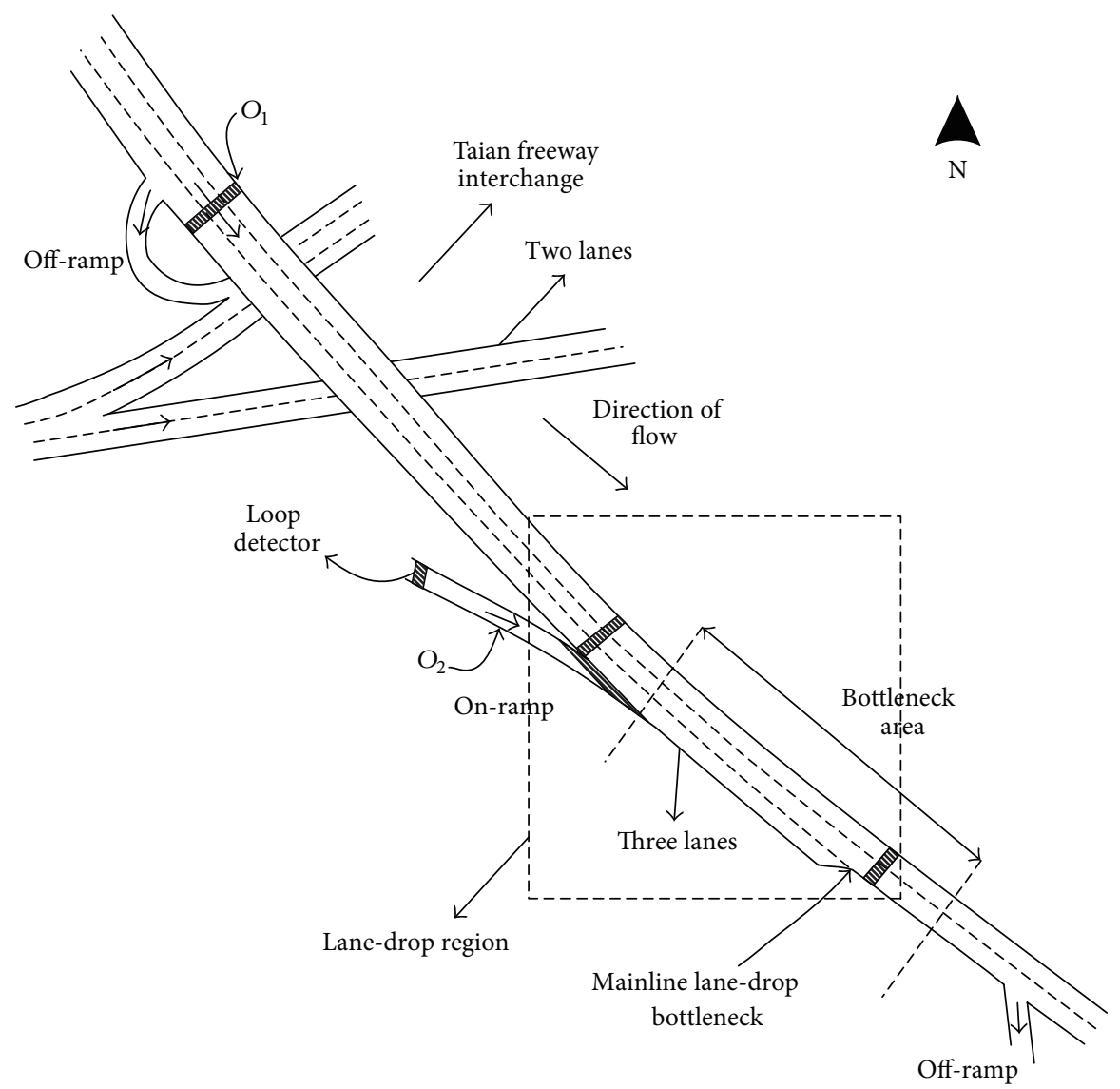

FIGURE 6: Study site of freeway network.

travels into the VSL region, which will lead to a poor VSL control. Accordingly, it is crucial to create section $B$ with adequate length $(500 \sim 700 \mathrm{~m})$ immediately upstream of the problem region [1]. The test freeway network of the G3 Jingtai freeway in China, which is about $2.75 \mathrm{~km}$ long, is considered for an in-depth analysis and evaluation of the efficiency and suitability of the integrated control strategy regarding the mainline land-drop bottleneck. There are five traffic loop detectors and an on-ramp on this test freeway network. There is a static posted speed limit of $100 \mathrm{~km} / \mathrm{h}$. The freeway test-bed is shown in Figure 6. This test freeway network always has recurrent heavy congestion during the afternoon peak hours due to high traffic demand, which then leads to an active bottleneck.

The freeway test network consists of speed limit area, a metered on-ramp, and a bottleneck region. The basic mainline links have three lanes and the on-ramp includes only a single lane. The data used for feeding and calibrating the model were collected from the loop detectors on the mainline and on-ramp of the test road of the G3 freeway networks on 25 April 2014. The density can be determined by analyzing the fundamental relations of the traffic variables. Ten seconds was selected as the discrete time interval. Collected data include the following information: flow, average speed, and occupancy. The traffic flow component is further calibrated using video data from the freeway mainline.

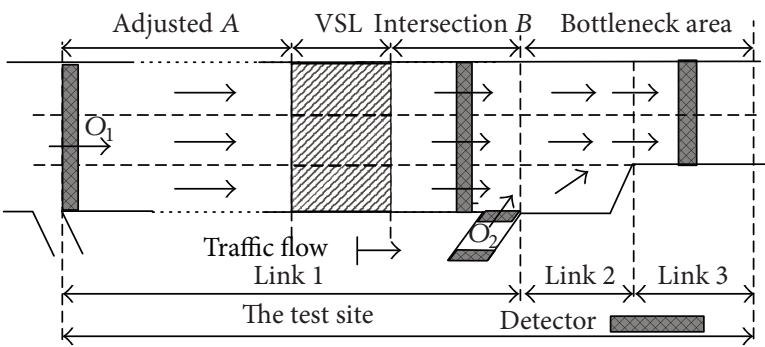

FIgURE 7: Freeway detectors layout.

According to the integrated control model demands, some detectors are placed on the test road as shown in Figure 7. Particularly, the freeway mainline bottleneck has two lanes with a capacity of $3500 \mathrm{veh} / \mathrm{h}$. The regular mainline links include three lanes with a capacity of $1750 \mathrm{veh} / \mathrm{h}$ for each lane. The network critical density and maximum density are $33.5 \mathrm{veh} / \mathrm{km} / \mathrm{l}$ and $180 \mathrm{veh} / \mathrm{km} / \mathrm{l}$, respectively. The lengths of network links are set to $1500 \mathrm{~m}, 750 \mathrm{~m}$, and $500 \mathrm{~m}$ for link 1 , link 2, and link 3, respectively. The lengths of the mainline and on-ramp segments are determined and set to 250 meters and 150 meters, respectively. The period of one VSL value generation and the controller sampling time interval are set to 90 seconds. 

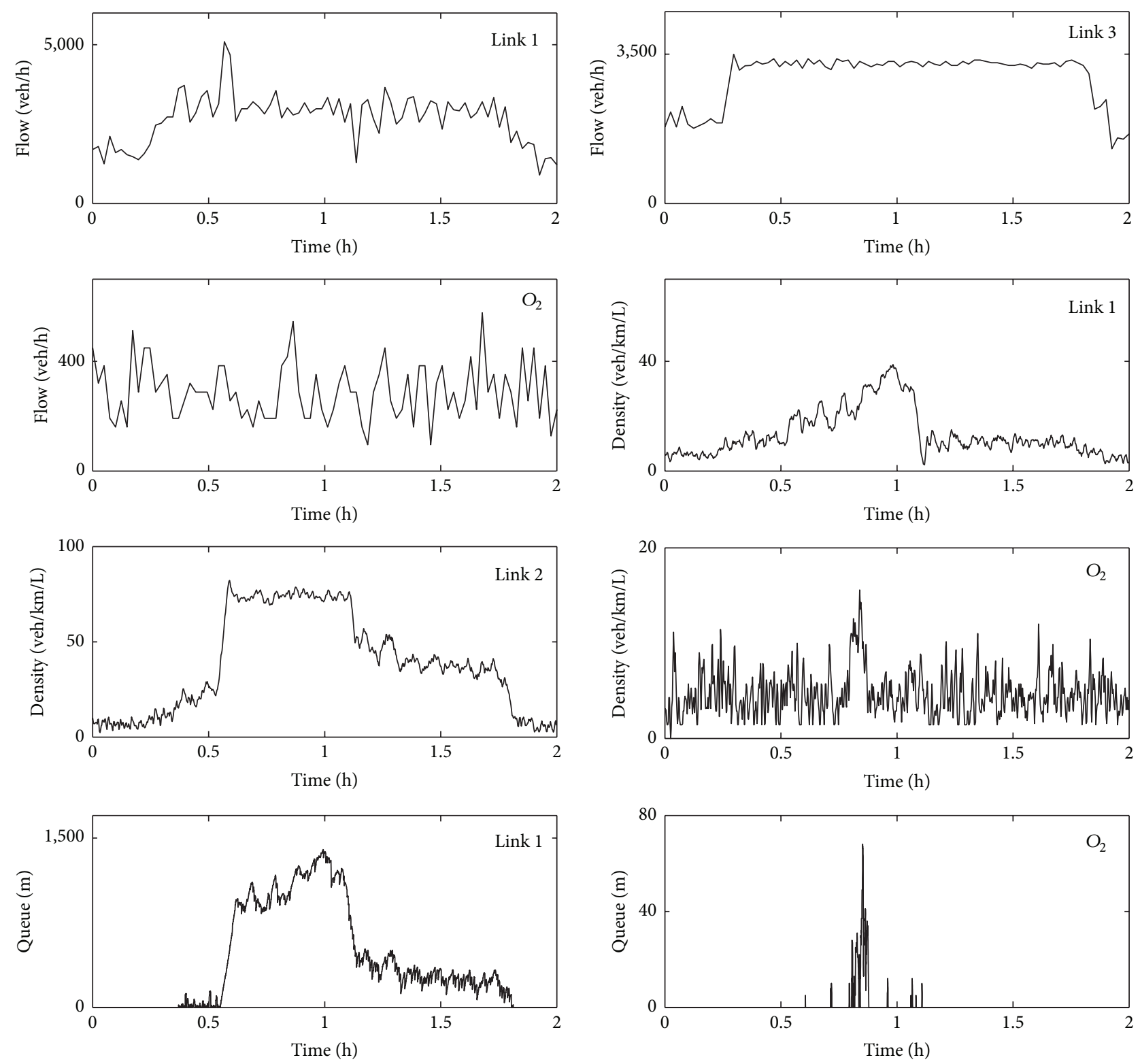

FIgURE 8: Traffic flow parameters under the no-control case.

As mentioned above, this paper explains the rationale behind introducing the model parameters $\alpha_{V}$ and $\alpha_{D}$. The values of these parameters are set to 1 and 50, based on their different dimensions. Specifically, if the weighting parameter $\alpha_{V}$ of the TTV model is set to 1 , the weight coefficient $\alpha_{D}$ of the TTD model is equal to 50. The simulation of approach application in this paper has been run over a time horizon of two hours, which corresponds to the length of an afternoon peak hour session. In this section we show the results of the simulations under three different conditions. To evaluate the above control schemes, some traffic flow parameters concerning the detected road have been achieved through simulation platform detectors, which are then processed by the program. The evaluation results are presented as described in the following sections.
5.1.1. No-Control Case. Figure 8 shows the resulting traffic flow, density, and queue profiles for partially typical links on a tested road when no control measure is applied. Since the legal speed limit value is adopted in case 1 (no-control case), traffic on the simulated system obeys self-organization. During the first 0.3 hours, the origin flow continuously increases, suggesting it has not yet reached capacity. The traffic in the free-flow condition and mainline link 1 has enough space to receive the outflow from on-ramp $\mathrm{O}_{2}$. During this period, the vehicles from mainline link 1 and onramp $\mathrm{O}_{2}$ have struggled for the right to travel downstream. The flow in link 3 of the bottleneck region reaches the actual capacity at $t=0.3 \mathrm{~h}$. As the arriving flow continues to increase, the traffic density on the mainstream or on-ramp increases as well; this leads to a set of congestion phenomena after $0.4 \mathrm{~h}$ 
TABLE 1: Delay under the no-control case.

\begin{tabular}{|c|c|c|c|c|c|}
\hline \multicolumn{3}{|c|}{ Total delay } & \multicolumn{3}{|c|}{ Average delay } \\
\hline Mainline delay & On-ramp delay & System delay & Mainline delay & On-ramp delay & System delay \\
\hline $530,764 \mathrm{veh} \cdot \mathrm{s}$ & 59,922 veh·s & $590,686 \mathrm{veh} \cdot \mathrm{s}$ & $74.2 \mathrm{~s}$ & $73.3 \mathrm{~s}$ & $74.1 \mathrm{~s}$ \\
\hline
\end{tabular}
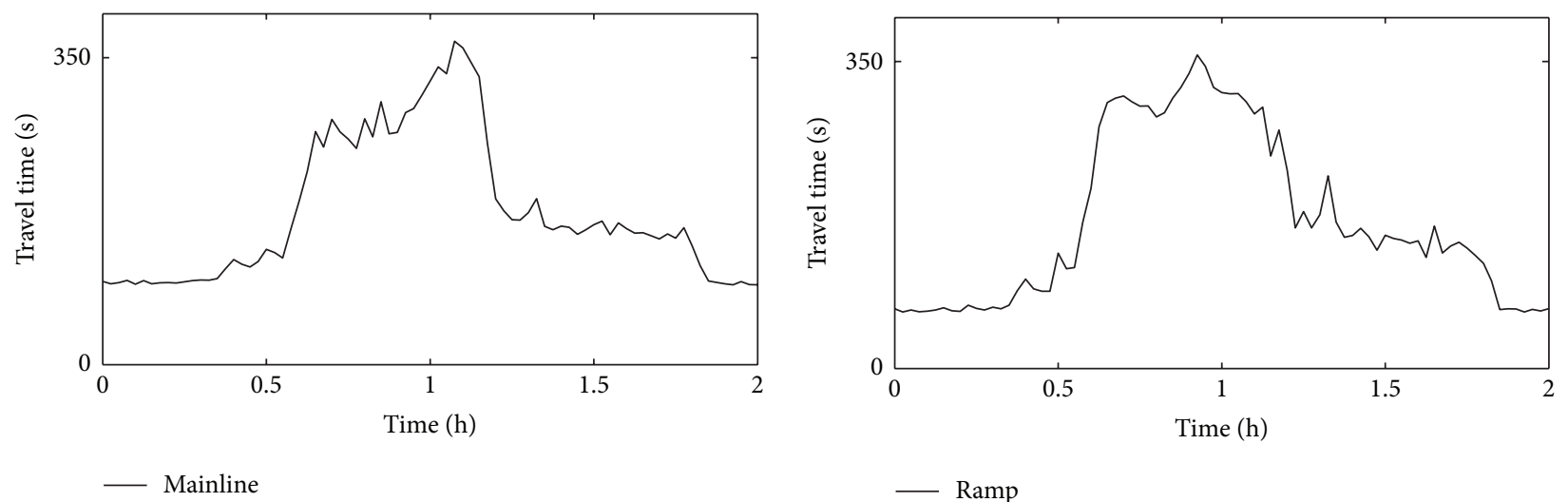

FIGURE 9: Travel time of the mainline and ramp under the no-control case.

in the simulated area. In particular, when no control measure is applied for the expressed demand scenario, congestion appears at the mainline section during peak hours that then propagates backwards all the way to the merge area $B$, where a queue is formed. Then the queue forming at link 2 in the bottleneck region reaches the merge area $B$, and a certain delay exists, which leads to a decreased outflow of on-ramp traffic. The congestion within the bottleneck region and the existing traffic disturbance result in a visible decrease around the bottleneck flow in link 3 .

With the origins $\mathrm{O}_{1}$ and $\mathrm{O}_{2}$ traffic flow being higher than the capacity of bottleneck link 3 , the observed congestion of the simulation network can be seen in Figure 8. Along with the origin inflow increasing, the traffic conditions turn from free-flow to jam. During the time period $t=0.4 \mathrm{~h}$ to $t=$ $1.8 \mathrm{~h}$, the queue length of mainline link 1 reached a maximum $1382.9 \mathrm{~m}$; a short queue (the average queue length is $16 \mathrm{~m}$ ) forms at the beginning of this period at on-ramp $\mathrm{O}_{2}$, because the bottleneck region has a certain place to meet the needs of the mainline queue. This congestion created in the bottleneck region travels upstream and impacts merge area $B$ at around $t=0.5 \mathrm{~h}$, leading to a visible traffic density increase as well as a speed decrease in both the bottleneck region link 2 and the mainline link 1 .

Figure 9 shows the average travel time of freeway mainline vehicles from the origin $O_{1}$ to the bottleneck link 3 and the on-ramp vehicles traveling from on-ramp $O_{1}$ to the bottleneck region during the total simulated time. By combining this piece of information with that of Figures 8 and 9 and Table 1, it becomes clear that during the peak period vehicles from the on-ramp joined the mainstream resulting in aggravated congestion on the mainline. Therefore, the congested traffic flow at an active bottleneck region results in an increased average travel time for both on-ramp and mainline vehicles amounting to $174 \mathrm{~s}$ and $168 \mathrm{~s}$, respectively.
Meanwhile, the average delays are equal to $74.2 \mathrm{~s}$ and $73.3 \mathrm{~s}$ in mainline and on-ramp, respectively. The resulting total delay is $590686 \mathrm{veh} \cdot \mathrm{s}$.

5.1.2. VSL Control. In case 2, the ramp metering is not taken into account. Instead, only the VSL control is applied to mainline link 1 . The mainstream link 1 is divided into three clusters with different characteristics (see Figure 7). The first cluster is mainly described as the intersection area $B$ with an outflow of the on-ramp $\mathrm{O}_{2}$ disturbed mainstream regular operation. The variable speed limits control is applied in the second cluster while the last cluster $A$ provides a place for the queue that is caused by the downstream VSL control. Figure 10 shows the relationship between the queues of link 1 and link 2, as well as the traffic flow and density profiles for the detected road. The travel time and the delay time are displayed in Figure 11 and Table 2, and the optimal VSL values trajectories are shown in Figure 12.

The situation here is actually identical to the no-control case until around $t=0.4 \mathrm{~h}$, because no congestion occurs until that point. At around $t=0.4 \mathrm{~h}$, the capacity of link 3 experiences a decrease phenomenon; however, its descending range is less than that of case 1 due to the VSL control that is applied in the mainline, which results in a limited outflow from link 1 upstream. The VSL value of link 1 switches gradually to values around $60 \mathrm{~km} / \mathrm{h}$, allowing the bottleneck region to fit a higher number of vehicles and thereby reducing the real loss in capacity. Since VSL control is applied on link 1 and the ramp metering is not taken into account, the traffic density on link 1 is higher while link 2 traffic density is lower than those same links in the no-control case.

The issue here is the need to keep the density of the bottleneck region close to its critical density and avoid congestion appearing after $t=0.4 \mathrm{~h}$ as it does in case 1 scenario. Moreover, when the mainline control strategy is applied it 

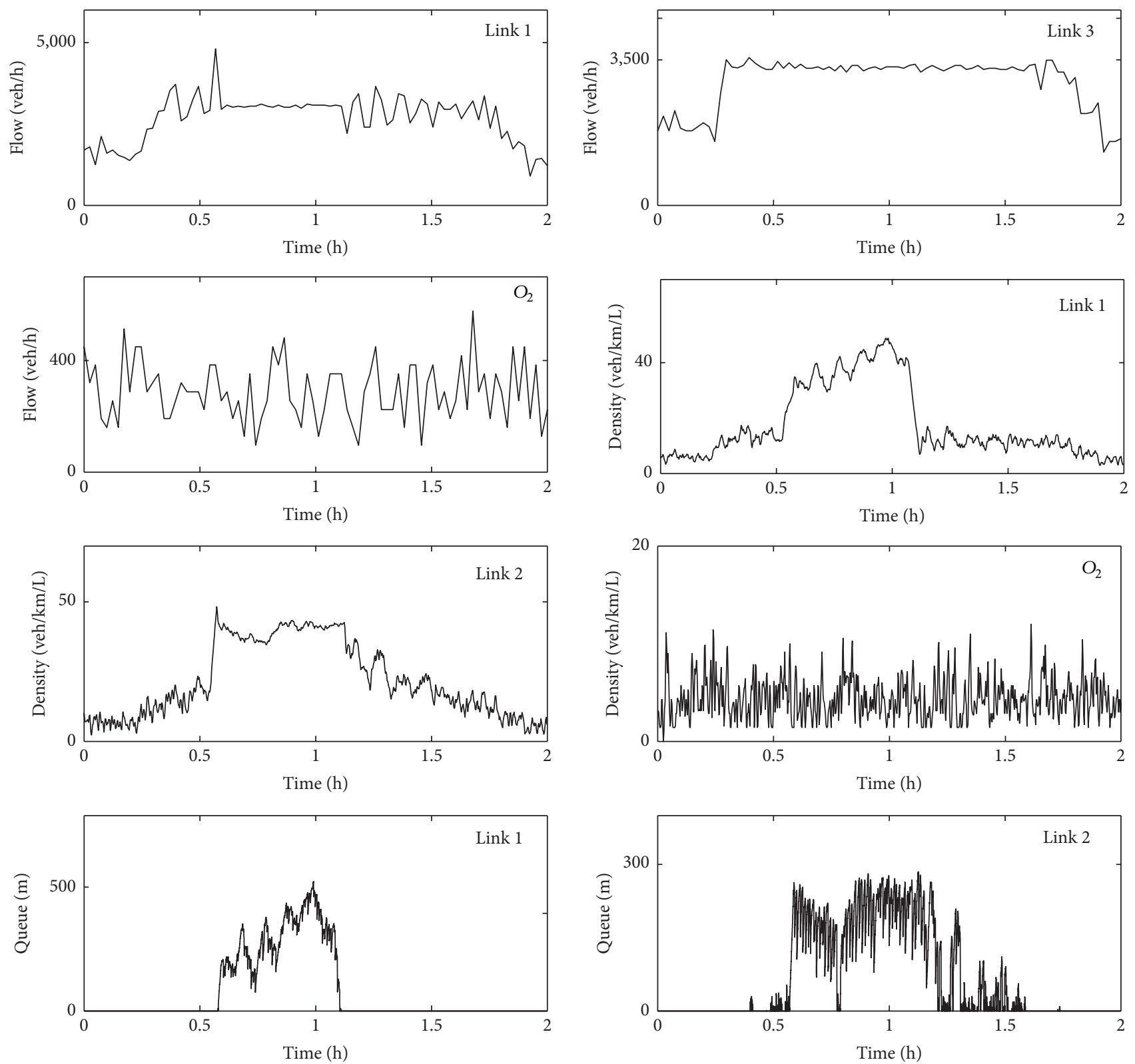

FIgURE 10: Traffic flow parameters under the VSL control case.
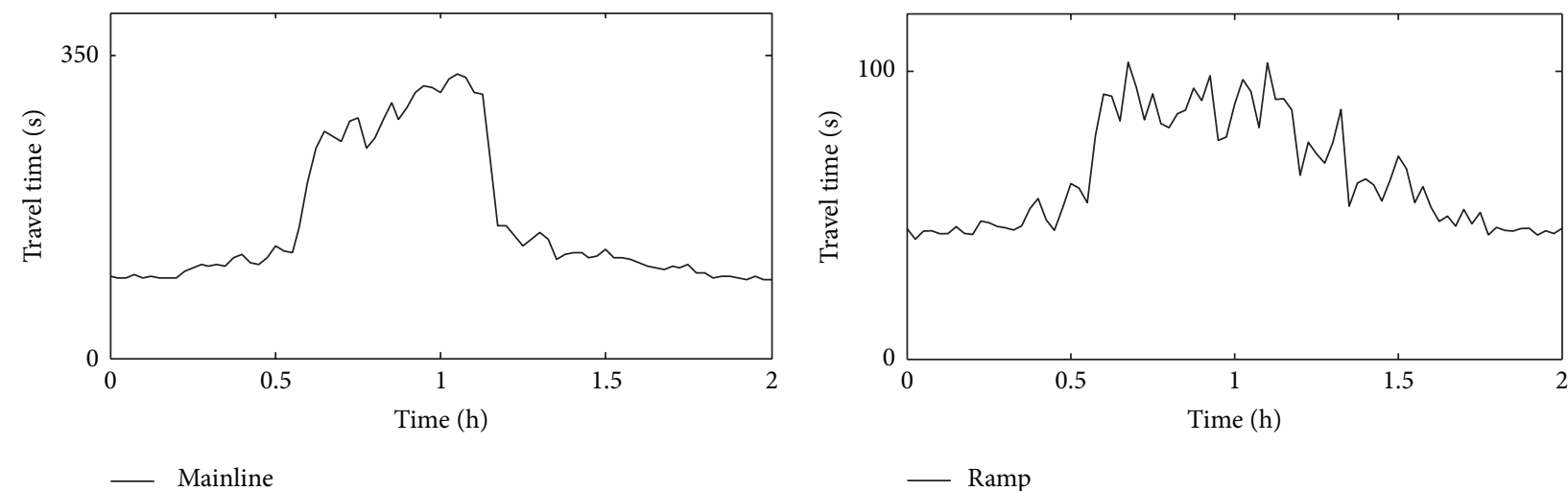

FIgURE 11: Travel time of the mainline and ramp under the VSL control case. 
TABLE 2: Delay under the VSL control case.

\begin{tabular}{lcccc}
\hline Mainline delay & $\begin{array}{c}\text { Total delay } \\
\text { On-ramp delay }\end{array}$ & System delay & Mainline delay & $\begin{array}{c}\text { Average delay } \\
\text { On-ramp delay }\end{array} \quad$ System delay \\
\hline $505,055 \mathrm{veh} \cdot \mathrm{s}$ & $20,287 \mathrm{veh} \cdot \mathrm{s}$ & $525,342 \mathrm{veh} \cdot \mathrm{s}$ & $70.6 \mathrm{~s}$ & $24.8 \mathrm{~s}$ \\
\hline
\end{tabular}

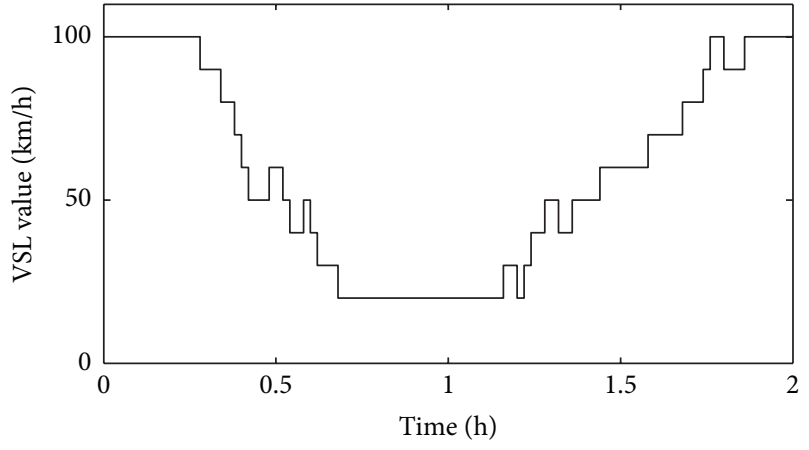

FIGURE 12: VSL values under the VSL control case.

should enable the maximum bottleneck region outflow. The VSL values for link 1 switch dynamically depending on how traffic parameters change, aiming to keep the bottleneck flow more stable. These temporary flow reductions are set up to hold the traffic density of link 2 close to critical density. However, after $t=0.6 \mathrm{~h}$, the VSL control has exhausted its energy and congestion in the bottleneck region becomes unavoidable.

In the mainline VSL control case, the optimal result is found for the mainstream. The total length of the mainline queue including link 1 and link 2 in case 2 is less than that of case 1 , which is due to the mainline traffic flow operating with more stability and offering enough right to traffic from $\mathrm{O}_{2}$, the latter of which explains why on-ramp $\mathrm{O}_{2}$ went without the queue phenomenon in case 2 . The queue length of link 2 in the bottleneck region is created during peak hours due to the heavy outflow from the on-ramp $\mathrm{O}_{2}$. Therefore, taking only the mainline VSL control could not fully meet the demands of the bottleneck flow control. The resulting total delay of both the mainline and on-ramp is still $525342 \mathrm{veh} \cdot \mathrm{s}$, which is only an $11 \%$ decrease compared to case 1 . Although the speed limit values reach the minimum value, the queue in the bottleneck region is heavier, because the outflow from on-ramp $\mathrm{O}_{2}$ continues to travel into the bottleneck region. Therefore, there is no queue on the on-ramp $\mathrm{O}_{2}$ in case 2. The average delay reaches $24.8 \mathrm{~s}$, which is influenced by the bottleneck region queue. The travel time for on-ramp vehicles is lower than those in no-control case. In general, the reason why VSL is not enough to solve congestion is that the controller operates at the lower bound.

Remarkably, in comparing case 2 with the no-control case, it can be seen that, in the mainline control case, the total length of the detected road queue is shorter, the delay is greatly decreased, and the traffic flow operation is more stable (meaning the density is more uniform). At the same time, however, the queue length on the mainline is longer, reaching $663 \mathrm{~m}$ at its peak.
5.1.3. Integrated Control. In the integrated control scenario, both ramp metering and mainline VSL control measures are possible. The control strategy applied in this case handles both control measures simultaneously, cooperating to a common purpose to collectively achieve maximum of the TTV and minimum of the TTD. Particularly, the mainline VSL control is chosen to operate on link 1 and the control region is founded at the same location as in case 2 because the variable speed limits control has the largest effect in this region. The VSL values can be seen in Figure 15.

The optimal control strategy is determined by the degree of congestion on the mainline and the on-ramp to maximum of the TTV and minimum of the TTD. As can be seen from Figure 13, there is no congestion in link 1 before the flow reaches capacity at around $t=0.6 \mathrm{~h}$, while the on-ramp queue is slightly increased due to the ramp metering actions. The outflow of the bottleneck region remains maximized because of the integrated control, which results in a lack of congestion in the bottleneck region. The optimal control strategy, which increases the possibilities to contain the abundant flow towards link 2, and the density of link 3 can be maintained close to a critical density value at around $t=$ $0.6 \mathrm{~h}$, after which unavoidable congestion occurs in link 1 and $\mathrm{O}_{2}$. In fact, the traffic density of link 2 is maintained close to the critical value of link 3 , which is necessary to maximize the freeway outflow. This leads to decrease in the TTD.

The observed higher outflow from the bottleneck region or link 3 at the time around $t=0.5 \mathrm{~h}$ is due to the application of a reasonable, optimal control strategy, which integrates the releasing rate of the flow from the upstream link 1 and onramp $\mathrm{O}_{2}$. According to Figure 13, the density of on-ramp $\mathrm{O}_{2}$ experiences some fluctuations during the time period from $t=0.6 \mathrm{~h}$ to $t=1.1 \mathrm{~h}$, because the density of the upstream link 1 appears higher than the traffic density of the on-ramp $\mathrm{O}_{2}$ so that the upstream vehicles (in link 1) achieve more access to cross the bottleneck region. Through adjusting the release rates of the on-ramp and the VSL values according to the degree of congestion of the mainline and the onramp, the queue situations on link 1 and the on-ramp $\mathrm{O}_{2}$ are efficiently controlled. Specifically, a higher traffic density of the upstream of link 1 corresponds to a higher VSL value and a lower releasing rate of the on-ramp. If the degree of congested traffic on the on-ramp is higher than that of link 1 , the releasing rate of the on-ramp traffic should be reasonably enhanced. The variables of the upstream of link 1 and the onramp $\mathrm{O}_{2}$ outflow are shown in Figure 13. When compared with case 1, the flow through the bottleneck region is larger before the time $t=1 \mathrm{~h}$, because the inflow of the bottleneck region is derived from both the upstream of link 1 and the on-ramp $\mathrm{O}_{2}$ which is close to the capacity of link 3 , which makes the flow of the bottleneck region more stable and reliable under the integrated control. However, the outflow 

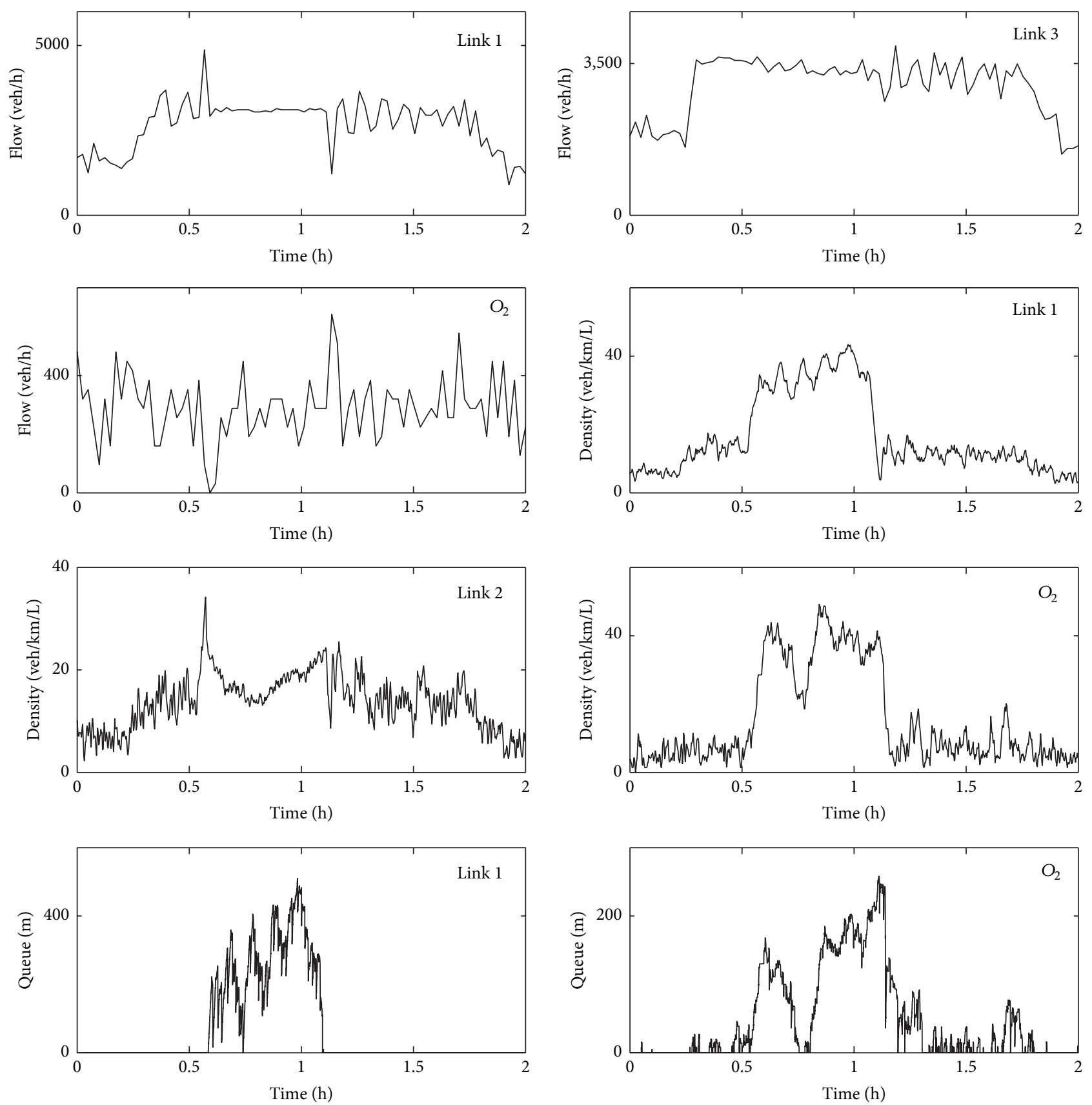

FIGURE 13: Traffic flow parameters under the integrated control case.

of link 3 curves, because the inflow of bottleneck region is below the capacity of link 3 . The outflow of the bottleneck region is equal to the arriving demand of the upstream, which fluctuates strongly in time and space during time period from $t=1.1 \mathrm{~h}$ to $1.7 \mathrm{~h}$. During the time period from $t=0.3 \mathrm{~h}$ to $t=$ $0.7 \mathrm{~h}$, the VSL values in case 3 remain higher than case 2 . After the time $t=1.1 \mathrm{~h}$, the speed limit values have a distinguished increase compared to case 2 .

The delay time is displayed in Table 3 . When the integrated control is applied, the TTD of both the mainline and on-ramp flow reach $433480 \mathrm{veh} \cdot \mathrm{s}$ and $63991 \mathrm{veh} \cdot \mathrm{s}$, respectively. The system average delay is equal to $62.4 \mathrm{~s}$, which shows a $15.78 \%$ and $5.31 \%$ improvement in case 1 and case 2, respectively. The total travel time of the detected road with the application of the integrated control equals $497471 \mathrm{veh} \cdot \mathrm{s}$, which corresponds to two improvements of $15.79 \%$ and $5.31 \%$ in case 1 and case 2, respectively. The average VSL value is higher than the average VSL value of case 2, because of coordination with the ramp metering in case 3 , whose releasing rates can be seen in Figure 16. According to Figure 14, the travel time curve under the integrated control condition in the upstream of link 1 is shown as a series of lower values than those found in previous cases, which resulted from the application of a reasonable control measure. Nevertheless, the 
TABle 3: Delay under the integrated control case.

\begin{tabular}{|c|c|c|c|c|c|}
\hline \multicolumn{3}{|c|}{ Total delay } & \multicolumn{3}{|c|}{ Average delay } \\
\hline Mainline delay & On-ramp delay & System delay & Mainline delay & On-ramp delay & System delay \\
\hline $433,480 \mathrm{veh} \cdot \mathrm{s}$ & 63,991 veh·s & $497,471 \mathrm{veh} \cdot \mathrm{s}$ & $60.6 \mathrm{~s}$ & $78.3 \mathrm{~s}$ & $62.4 \mathrm{~s}$ \\
\hline
\end{tabular}

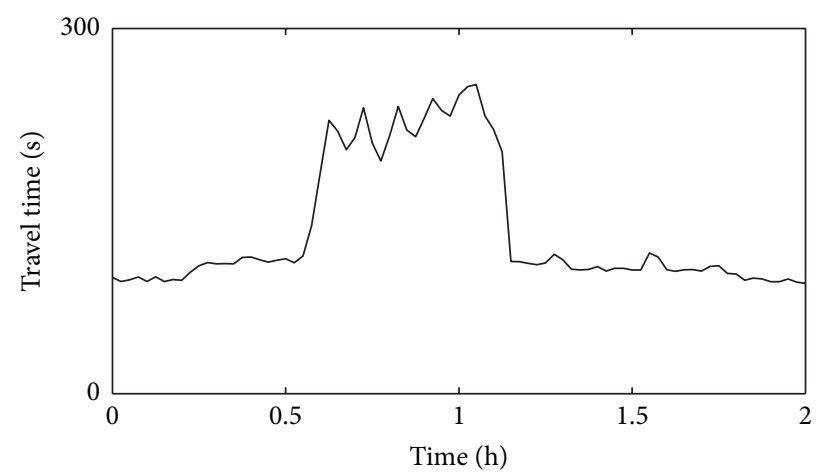

_ Mainline

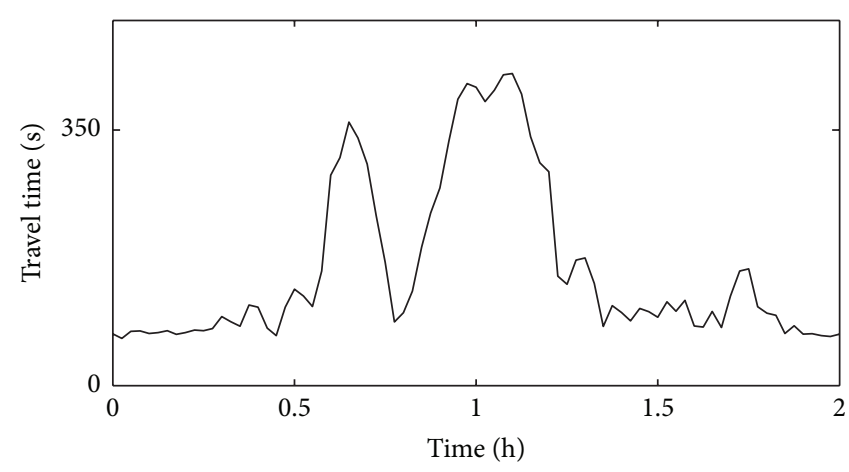

— Ramp

Figure 14: Travel time of the mainline and ramp under the integrated control case.

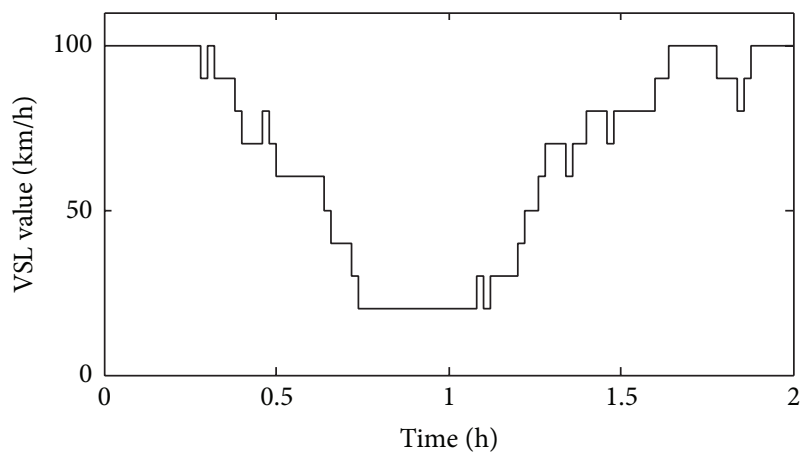

FIGURE 15: VSL values under the integrated control case.

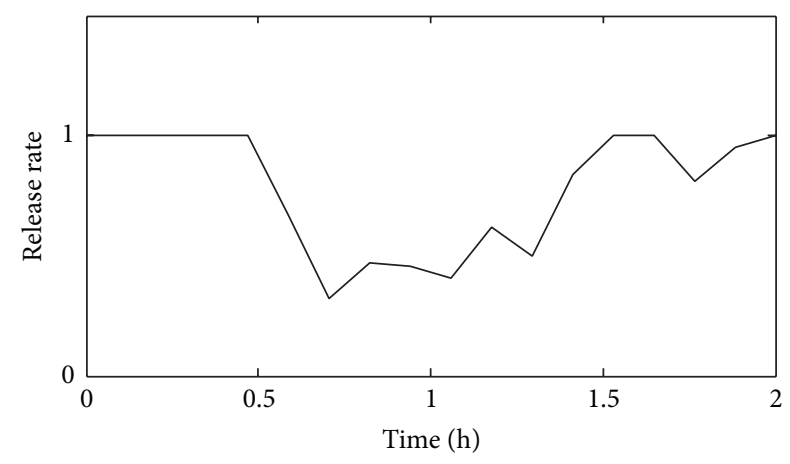

FIGURE 16: Metering rates for on-ramp.

on-ramp travel time continues to experience higher values during peak hours, which is due to the fact that the on-ramp outflow is restricted by the adjustment of the releasing rates. As a result, the average delay time of the on-ramp $\mathrm{O}_{2}$ is longest compared with other cases, which is equal to $78.3 \mathrm{~s}$. This means that the integrated control strategy is an efficient way to control the upstream of link 1 and the on-ramp $\mathrm{O}_{2}$, since it considers both the degrees of congestion on the upstream of link 1 and $\mathrm{O}_{2}$ to control the outflow from link 1 and $\mathrm{O}_{2}$ by adjusting the VSL values and the metering rates.

\section{Conclusions}

The paper presented an optimal integrated control strategy for freeway mainline lane-drop bottleneck regions which includes the variable speed limits and ramp metering. This idea was illustrated by a simple freeway lane-drop bottleneck network, where the various cases including no-control, mainline VSL control, and integrated control were compared for the total delay and outflow of the bottleneck region. This paper then offered a detailed discussion of three different cases aimed at demonstrating the efficiency and the flexibility of integrated freeway bottleneck traffic control. In the VSL control case, variable speed limits control was proven to be effective when compared to the no-control case. When only the VSL control was applied to the bottleneck upstream, there was no queue on the on-ramp and the bottleneck traffic congestion was instantly relieved. However, the queue phenomenon was serious on the upstream mainline. Furthermore, we adopted the integrated control measures to solve the traffic congestion problem and enhance the performance in the freeway network. The integrated control on freeway included a reasonable set of VSL values and ramp release rates, depending on the degrees of congestion in the integrated bottleneck upstream mainline and on the on-ramp. We found that the integrated control case results in the bottleneck region had a higher outflow and a significantly lower total delay when providing a certain right to the ramp outflow.

Although the optimal integrated freeway traffic control measures can dynamically manage bottleneck region congestion and reasonably control the degrees of both the on-ramp and upstream, the real-life benefits of optimal integrated 
freeway traffic control strategy have yet to be applied. In the future, the integrated control measures of combining the mainline VSL and ramp metering should be applied to and tested on real freeways. Another interesting and relevant issue for future study is the research of complexes freeway network, such as dynamic traffic route guidance, ring road around cities, and regions where some cities are connected by freeway. Some other control approaches also should be considered in addition to variable speed limits and ramp metering (such as drivers' information, peak-lanes, and tidal traffic flow).

\section{Disclaimer}

These viewpoints expressed in this paper belong to the authors and do not constitute a standard, specification, or regulation.

\section{Conflict of Interests}

The authors declare that there is no conflict of interests regarding the publication of this paper.

\section{Acknowledgments}

This research was supported by the Intelligent Transportation Systems (ITS) Research Center, College of Transportation, Jilin University, in China and was partly funded by the National High Technology Research and Development Program of China (no. 2014BAG03B03).

\section{References}

[1] M. Hadiuzzaman and T. Z. Qiu, "Cell transmission model based variable speed limit control for freeways," Canadian Journal of Civil Engineering, vol. 40, no. 1, pp. 46-56, 2013.

[2] S. Smulders, "Control of freeway traffic flow by variable speed signs," Transportation Research Part B: Methodological, vol. 24, no. 2, pp. 111-132, 1990.

[3] C. Feng, J. Yuanhua, N. Zhonghai, Y. Huixin, and S. Huijuan, "Ramp metering research of junction based on fuzzy neural network model," Journal of Transportation Systems Engineering and Information Technology, vol. 11, no. 1, pp. 108-113, 2011.

[4] A. Kotsialos and M. Papageorgiou, "Efficiency and equity properties of freeway network-wide ramp metering with AMOC," Transportation Research Part C: Emerging Technologies, vol. 12, no. 6, pp. 401-420, 2004.

[5] H. L. Khoo, "Dynamic penalty function approach for ramp metering with equity constraints," Journal of King Saud University-Science, vol. 23, no. 3, pp. 273-279, 2011.

[6] S. Yousif and J. Al-Obaedi, "Modeling factors influencing the capacity of motorway merge sections controlled by ramp metering," Procedia-Social and Behavioral Sciences, vol. 16, pp. 172-183, 2011.

[7] F. Middelham, "A synthetic study of the network effects of ramp metering," Tech. Rep., Transport Research Center (AVV), Dutch Ministry of Transport, Public Works and Water Management, 1999.

[8] I. Papamichail, A. Kotsialos, I. Margonis, and M. Papageorgiou, "Coordinated ramp metering for freeway networks-a model-predictive hierarchical control approach," Transportation Research C: Emerging Technologies, vol. 18, no. 3, pp. 311-331, 2010.

[9] I. Papamichail and M. Papageorgiou, "Balancing of queues or waiting times on metered dual-branch on-ramps," in Proceedings of the 13th International IEEE Conference on Intelligent Transportation Systems (ITSC '10), pp. 1448-1455, IEEE, September 2010.

[10] R. C. Carlson, I. Papamichail, M. Papageorgiou, and A. Messmer, "Optimal motorway traffic flow control involving variable speed limits and ramp metering," Transportation Science, vol. 44, no. 2, pp. 238-253, 2010.

[11] A. Hegyi, B. De Schutter, and H. Hellendoorn, "Model predictive control for optimal coordination of ramp metering and variable speed limits," Transportation Research Part C: Emerging Technologies, vol. 13, no. 3, pp. 185-209, 2005.

[12] A. Hegyi, B. D. Schutter, and J. Hellendoorn, "Optimal coordination of variable speed limits to suppress shock waves," IEEE Transactions on Intelligent Transportation Systems, vol. 6, no. 1, pp. 102-112, 2005.

[13] C. Lee, B. Hellinga, and F. Saccomanno, "Evaluation of variable speed limits to improve traffic safety," Transportation Research C: Emerging Technologies, vol. 14, no. 3, pp. 213-228, 2006.

[14] Z. Li, Y. Li, P. Liu, W. Wang, and C. Xu, "Development of a variable speed limit strategy to reduce secondary collision risks during inclement weathers," Accident Analysis and Prevention, vol. 72, pp. 134-145, 2014.

[15] M. Abdwl-Aty, R. J. Cunningham, V. V. Gayah, and L. Hsia, "Dynamic variable speed limit strategies for real-time crash reduction on freeways," Transportation Research Record, vol. 2078, pp. 108-116, 2008.

[16] B. G. Heydecker and J. D. Addison, "Analysis and modelling of traffic flow under variable speed limits," Transportation Research Part C: Emerging Technologies, vol. 19, no. 2, pp. 206-217, 2011.

[17] S. K. Zegeye, B. De Schutter, J. Hellendoorn, and E. A. Breunesse, "Variable speed limits for green mobility," in Proceedings of the IEEE Conference on Intelligent Transportation Systems (ITSK '11), pp. 2174-2179, 2011.

[18] L. Kejun, Y. Meiping, and Z. Jianlong, "Model predictive control for variable speed limit in freeway work zone," in Proceedings of the 27th Chinese Control Conference, pp. 488-493, July 2008.

[19] A. Nissan and H. N. Koutsopoulosb, "Evaluation of the impact of advisory variable speed limits on motorway capacity and level of service," Procedia-Social and Behavioral Sciences, vol. 16, pp. 100-109, 2011.

[20] X. Yang, Y. Lin, Y. Lu, and N. Zou, “Optimal variable speed limit control for real-time freeway congestions," Procedia-Social and Behavioral Sciences, vol. 96, pp. 2362-2372, 2013.

[21] R. Yu and M. Abdel-Aty, "An optimal variable speed limits system to ameliorate traffic safety risk," Transportation Research Part C: Emerging Technologies, vol. 46, pp. 235-246, 2014.

[22] R. C. Carlson, I. Papamichail, and M. Papageorgiou, "Integrated feedback ramp metering and mainstream traffic flow control on motorways using variable speed limits," Transportation Research Part C: Emerging Technologies, vol. 46, pp. 209-221, 2014.

[23] E. Grumert and A. Tapani, "Impacts of a cooperative variable speed limit system," Procedia-Social and Behavioral Sciences, vol. 43, pp. 595-606, 2012.

[24] N. Bhouri, H. Haj-Salem, and J. Kauppila, "Isolated versus coordinated ramp metering: field evaluation results of travel time reliability and traffic impact," Transportation Research C: Emerging Technologies, vol. 28, pp. 155-167, 2013. 
[25] A. Kotsialos, M. Papageorgiou, C. Diakaki, Y. Pavlis, and F. Middelham, "Traffic flow modeling of large-scale motorway networks using the macroscopic modeling tool METANET," IEEE Transactions on Intelligent Transportation Systems, vol. 3, no. 4, pp. 282-292, 2002.

[26] G. Gomes and R. Horowitz, "Optimal freeway ramp metering using the asymmetric cell transmission model," Transportation Research C: Emerging Technologies, vol. 14, no. 4, pp. 244-262, 2006.

[27] R. C. Carlson, D. Manolis, I. Papamichail, and M. Papageorgiou, "Integrated ramp metering and mainstream traffic flow control on freeways using variable speed limits," Procedia-Social and Behavioral Sciences, vol. 48, pp. 1578-1588, 2012.

[28] R. C. Carlson, I. Papamichail, M. Papageorgiou, and A. Messmer, "Optimal mainstream traffic flow control of large-scale motorway networks," Transportation Research Part C: Emerging Technologies, vol. 18, no. 2, pp. 193-212, 2010.

[29] M. Papageorgiou, J. Blosseville, and H. Haj-Salem, "Modeling and real-time control of traffic flow on the southern part of Boulevard Peripherique in Paris: part II: integrated on-ramp metering," Transportation Research Part A: General, vol. 24, no. 5, pp. 361-370, 1990.

[30] M. Papageorgiou, J. Blosseville, and H. Hadj-Salem, "Modeling and real-time control of traffic flow on the southern part of Boulevard Peripherique in Paris: part I: modeling," Transportation Research Part A: General, vol. 24, no. 5, pp. 345-359, 1990.

[31] A. Kotsialos, M. Papageorgiou, M. Mangeas, and H. Haj-Salem, "Integrated and integrated control of motorway networks via non-linear optimal control," Transportation Research Part C: Emerging Technologies, vol. 10, no. 1, pp. 65-84, 2002.

[32] A. Kotsialos, M. Papageorgiou, and A. Messmer, "Optimal integrated and integrated motorway network traffic control," in Proceedings of the 14th International Symposium on Transportation and Traffic Theory (ISTTT '99), pp. 621-644, Jerusalem, Israel, 1999.

[33] M. Hadiuzzaman, J. Fang, Y. Luo, and T. Z. Qiu, "Evaluating performance of a proactive optimal variable speed limit control using different objective functions," Procedia-Social and Behavioral Sciences, vol. 96, pp. 2895-2906, 2013. 


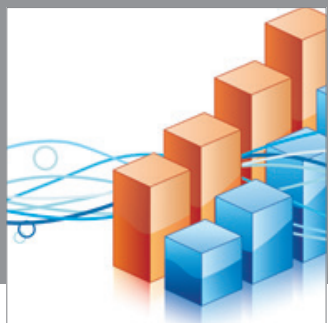

Advances in

Operations Research

mansans

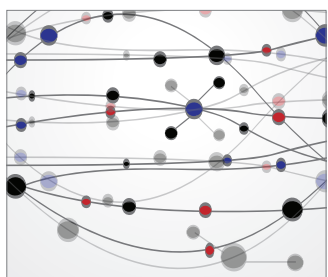

The Scientific World Journal
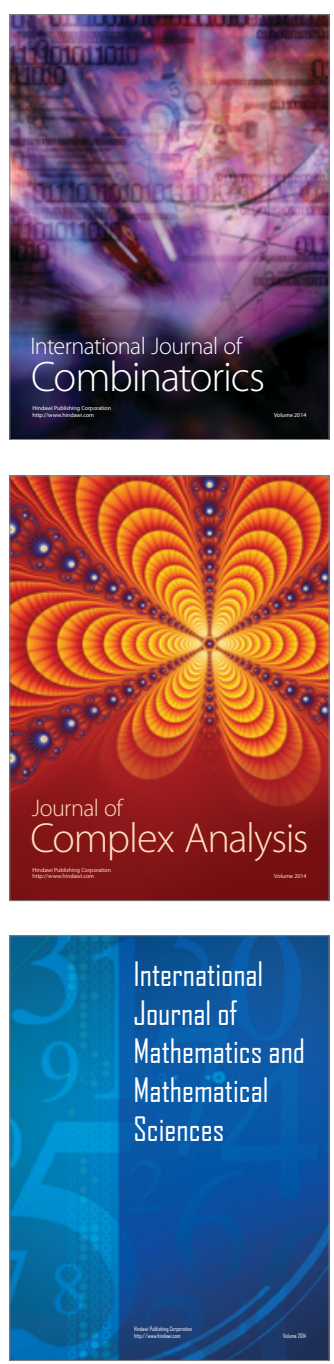
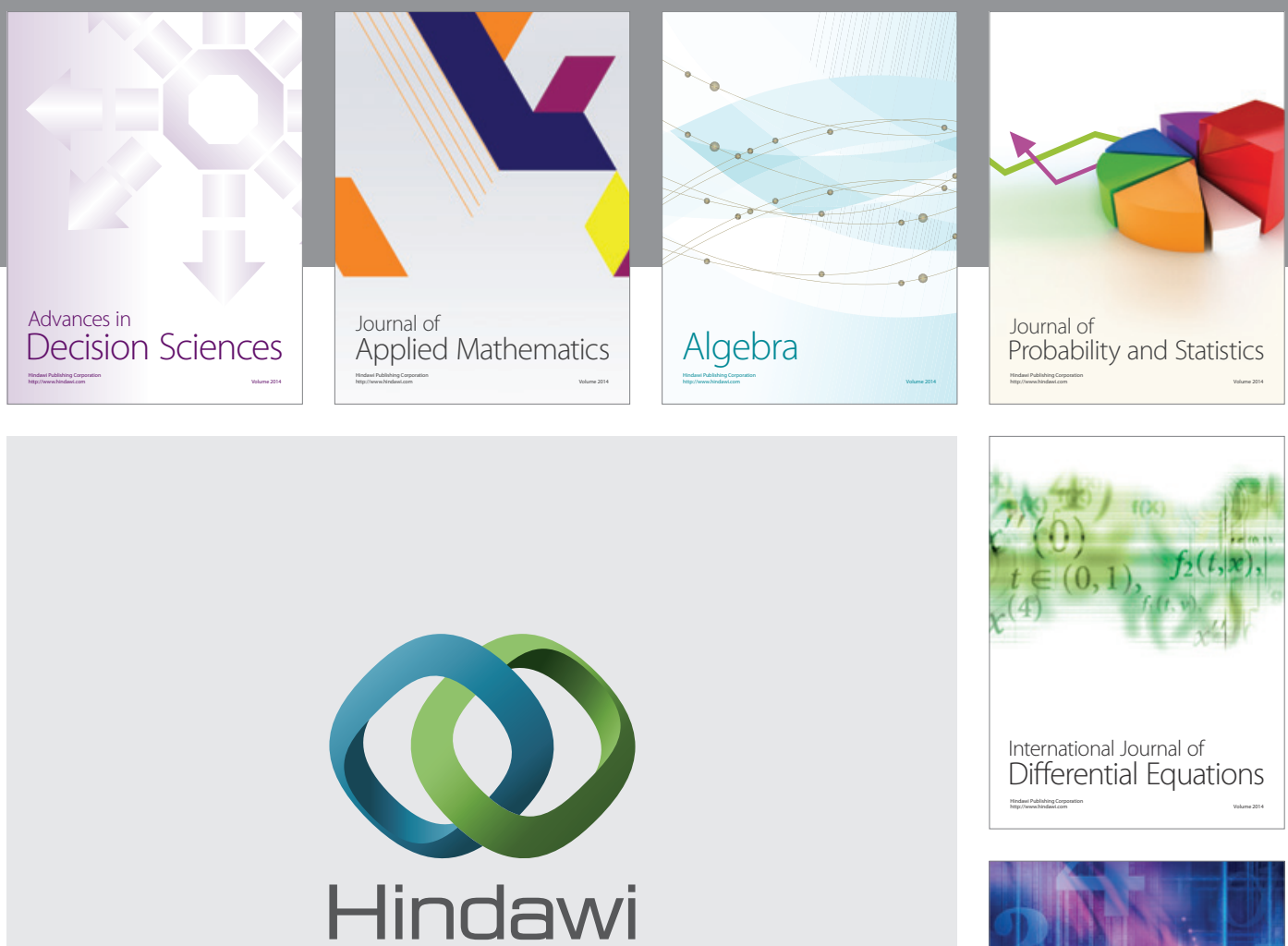

Submit your manuscripts at http://www.hindawi.com
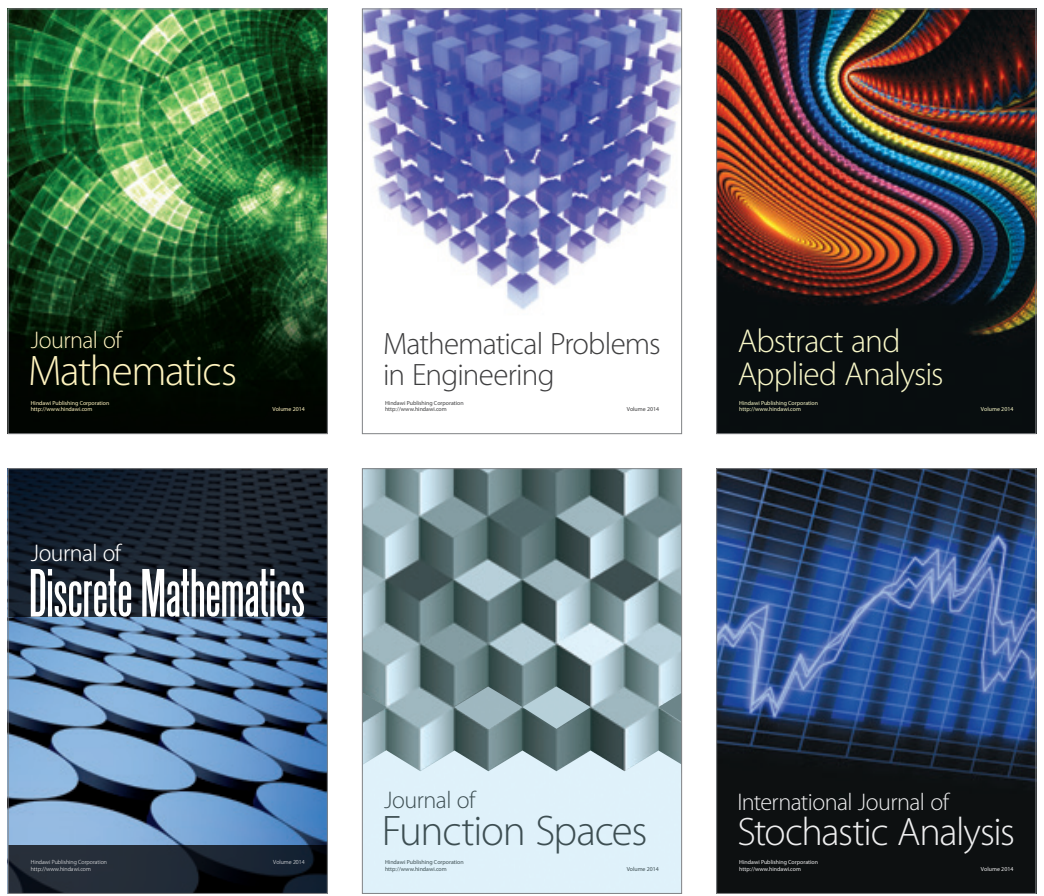

Journal of

Function Spaces

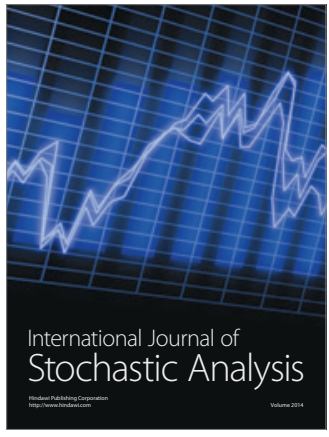

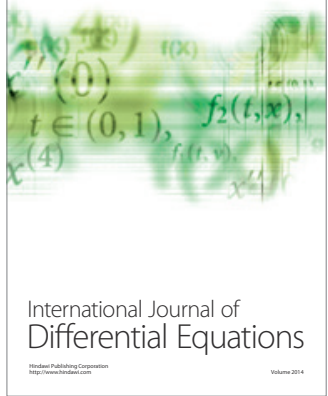
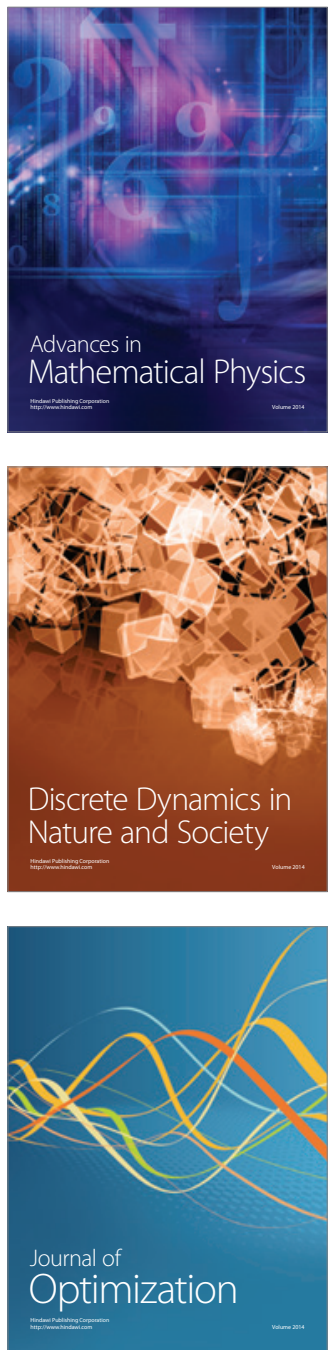